\title{
Review Article \\ Patient Data Prioritization in the Cross-Layer Designs of Wireless Body Area Network
}

\author{
Fasee Ullah, ${ }^{1}$ Abdul Hanan Abdullah, ${ }^{1}$ \\ Muhammad Qasim Jan, ${ }^{2}$ and Kashif Naseer Qureshi ${ }^{1}$ \\ ${ }^{1}$ Faculty of Computing, Universiti Teknologi Malaysia (UTM), 81310 Skudai, Johor, Malaysia \\ ${ }^{2}$ Department of Computing and Technology, Abasyn University, Peshawar 25000, Pakistan \\ Correspondence should be addressed to Abdul Hanan Abdullah; hanan@utm.my
}

Received 9 July 2015; Revised 28 September 2015; Accepted 30 September 2015

Academic Editor: Eduardo da Silva

Copyright (C) 2015 Fasee Ullah et al. This is an open access article distributed under the Creative Commons Attribution License, which permits unrestricted use, distribution, and reproduction in any medium, provided the original work is properly cited.

In Wireless Body Area Network (WBAN), various biomedical sensors (BMSs) are deployed to monitor various vital signs of a patient for detecting the abnormality of the vital signs. These BMSs inform the medical staff in advance before the patient's life goes into a threatening situation. In WBAN, routing layer has the same challenges as generally seen in WSN, but the unique requirements of WBANs need to be addressed by the novel routing mechanisms quite differently from the routing mechanism in Wireless Sensor Networks (WSNs). The slots allocation to emergency and nonemergency patient's data is one of the challenging issues in IEEE 802.15.4 and IEEE 802.15.6 MAC Superframe structures. In the similar way, IEEE 802.15.4 and IEEE 802.15.6 PHY layers have also unique constraints to modulate the various vital signs of patient data into continuous and discrete forms. Numerous research contributions have been made for addressing these issues of the aforementioned three layers in WBAN. Therefore, this paper presents a cross-layer design structure of WBAN with various issues and challenges. Moreover, it also presents a detail review of the existing cross-layer protocols in the WBAN domain by discussing their strengths and weaknesses.

\section{Introduction}

Every year, millions of people suffer from chronic disorders due to unavailability of health resources in time [1]. Due to poor health resources, wireless technologies have been stimulated with various tremendous changes in health sector, where BMSs are deployed to monitor different vital signs in a patient body [2]. BMSs are implantable (in-body), wearable (on-body), and/or installed away from the patient's body (offbody) to monitor various vital signs in a patient body such as EEG, ECG, EMG, heartbeat, respiratory rate, temperature, blood pressure, glucose level, mental status, RUN, and WALK [3-5]. These types of BMSs are connected wirelessly with a centralized device, that is, body area network coordinator (BANC) [3] or body area network (BAN) [6-9] as shown in Figure 1. Usually, patient data is classified into four classes, namely, critical data packet $(\mathrm{CP})$, reliability data packet (RP), delay data packet (DP), and ordinary data packet (OP) [10]. These four types of patient data are also known as nature of data in WBAN [11]. The CP data is the most critical data which comprises low threshold values of vital signs such as low heartbeat and low respiratory rate; therefore, the first slot (channel) is provided to transmit to the medical team for necessary action. The RP is the second type of critical patient data and contains high threshold values of vital signs such as high heartbeat and high respiratory rate. The DP is the noncritical data; therefore, it is placed in the third position in critical data ranking category. The DP data has an audio/video streaming of a patient for physical examination. $\mathrm{OP}$ is placed in the fourth position which contains routine data of the patient's body such as temperature reading. The BANC or BAN is the responsible device for allocating slots to BMSs on the basis of threshold values. The slots allocation is a challenging problem in WBAN because WBAN has faced unique constraints such as temperature-rise during monitoring of vital signs, detection of emergency data and allocation of slots to them on priority-basis, selection of appropriate paths to transmit data, limited signal strength of 


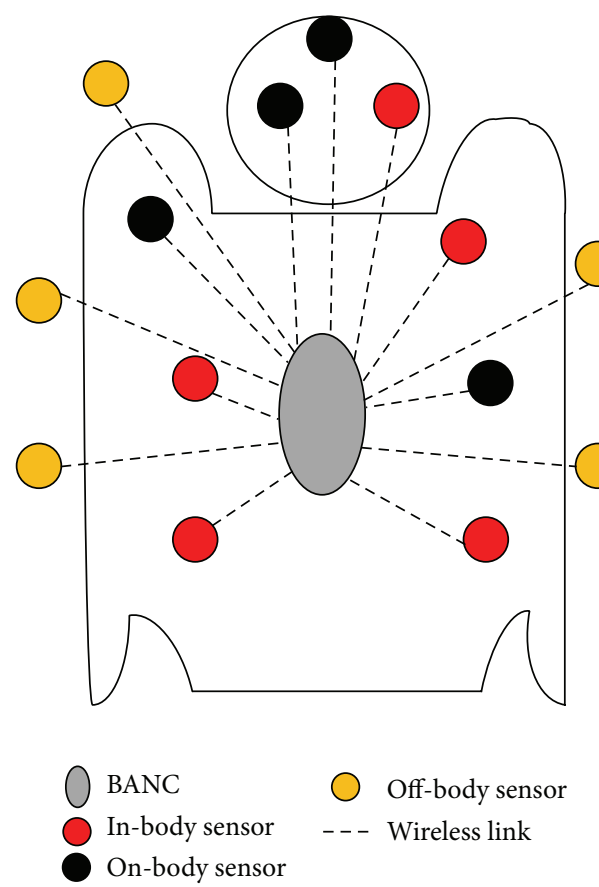

FIGURE 1: Human body covered with various wireless biomedical sensors.

an antenna, high energy consumption during selection and verification of temperature-aware paths for BMSs, updating routing table, mobility, and security $[4,12]$. These challenging problems require higher attention to solve them.

The cross-layer [13] is the only solution where all types of the required services are verified in routing layer, medium access control (MAC) layer, and PHY layer for BMSs in WBAN. Furthermore, the application and transport layer associate with routing, MAC, and PHY layer of TCP/IP protocol suites in order to establish a connection between all layers for data transmission [14]. The purpose of a cross-layer design is not to redesign the structure of TCP/IP protocol but to provide the interlayer communication services between different layers for exchanging of information between BMSs. Each layer shares different services such as data encryption, data format, session establishment, session maintenance, and dedicated allocation of slot (bandwidth) for the patient data depending on the requested services.

An overview of functionalities of each layer is provided in the cross-layer design for wireless communication. The application layer provides data format, compression, and encryption for end-to-end messages which keeps the message away from integrity violation during data transmission [15]. The transport layer divides continuous and huge amount of a patient data into different blocks of messages (data) in order to achieve high data reliability which protects the network from congestion during transmission such as huge data packet of ECG [15]. The network (routing) layer selects those BMSs that have minimum temperature and can carry data from source to the destination with minimum energy consumption. This type of communication provides reliable data transmission path [15]. Moreover, it also provides security to the messages during transmission with the support of IPSec protocol [16]. The MAC layer provides different scheduling access schemes such as contention-based slot allocation, priority-based slot allocation, and backoffs that are associated to IEEE 802.11, IEEE 802.15, and IEEE 802.16 families [15, 17, 18]. The PHY layer uses the same IEEE families' structure to provide various services such as transmission power strength, channel frequency modulation, and data rate adaption for wireless networks $[13,15,18]$. Numerous contributions have been made in the cross-layer design for WBAN. This paper presents various existing research contributions regarding WBAN in terms of mobility, topology, delay, energy consumption, classification of patient data, channel (slot) allocation to nonemergency and emergency data, temperature-rise, routing table, selection of the shortest path on the basis of reliability in terms of minimum temperature, and Packet Delivery Ratio (PDR). The purpose of cross-layer designs is to show how to investigate various problems in the routing, MAC, and PHY layers with their required services during data transmission.

The rest of the paper is constructed as follows: Section 2 presents various implantable and wearable BMSs with their functionalities. The information sharing in the intra- and interlayers of sensors in the cross-layers is classified into Manager, Nonmanager, centralized, and distributed methods, present in Section 3. Section 4 presents cross-layer design in terms of routing, MAC, and PHY layers for WBAN with their different functionalities. Section 5 presents various research contributions which have been made in cross-layers for routing, MAC, and PHY layers. Section 6 presents the comparative study of existing schemes. Finally, Section 7 presents conclusion of this paper.

\section{Classification of Patient's Body Sensors}

BMS comprises a physiological signal and radio transceiver [19]. The physiological sensor monitors different vital signs of a patient body and converts the monitored analog signals into digitized pattern. The digitized data is forwarded to radio transceiver which further transmits it to the BANC. The BANC forwards these data to the medical staff through Public Switch Telephone Network (PSTN) [24] for necessary actions against the vital signs conditions. There are two types of BMSs that monitor various vital signs of a patient's body, namely, wearable and implantable sensors as shown in Table 1 with their functionalities $[19,20]$. The wearable sensors are attached on skin of a human body or placed near human body where they monitor various vital signs such as blood pressure, EEG, ECG, EMG, and temperature. The implantable sensors are implanted inside the patient body and this implantation can be in the skin or tissues of a patient body. The examples of implantable BMSs are artificial retina, artificial cochlea, and camera pill. Table 1 describes data rate, signal types, and topology with functionalities of different wearable and implantable BMSs $[19,20]$.

BMSs are connected to BANC in the star topology and BANC is responsible for allocating channels to BMSs. This 
TABLE 1: BMSs functionalities in WBAN $[19,20]$.

\begin{tabular}{|c|c|c|c|c|c|}
\hline Sensor & Placement & Data rate & Signal type & Topology & Function \\
\hline Blood pressure & \multirow{8}{*}{ Wearable } & High & Discrete & \multirow{11}{*}{ Star } & Measures maximum and minimum threshold values \\
\hline EEG/ECG/EMG & & High & Continuous & & Measures voltage differences \\
\hline Humidity & & Very low & Discrete & & Observes humidity changes \\
\hline $\begin{array}{l}\text { Blood oxygen saturation } \\
\left(\mathrm{CaO}_{2}\right)\end{array}$ & & Very low & Discrete & & $\begin{array}{l}\text { Measures absorption ratio in blood oxygen } \\
\text { saturation }\end{array}$ \\
\hline Pressure & & High & Continuous & & Measures pressure values \\
\hline Respiration & & High & Continuous & & Measures breathing of the patient \\
\hline Glucose & & High & Discrete & & Measures the blood circulation rate in a body \\
\hline Temperature & & Very low & Continuous & & Measures the coolness or hotness of a body \\
\hline Artificial retina & \multirow{3}{*}{ Implantable } & High & Continuous & & $\begin{array}{l}\text { Collects information from the environment and } \\
\text { converts it into the electrical signals }\end{array}$ \\
\hline Artificial cochlea & & High & Continuous & & $\begin{array}{l}\text { Is implanted in ears and helps to convert voice } \\
\text { signals into pulses }\end{array}$ \\
\hline Camera pill & & High & Continuous & & $\begin{array}{l}\text { Swallows the pill in order to monitor various parts of } \\
\text { a body }\end{array}$ \\
\hline
\end{tabular}

is a challenging problem $[25,26]$ : how the sensor can use routing, MAC, and PHY layers for allocating the first slot on the priority-basis to emergency among heterogeneous nature of patient data? For example, the heartbeat sensor detects low threshold values (e.g., 20 beats/min) and the temperature sensor detects normal values. The vital sign of low threshold values is a life-threatening situation and it should be given the first priority to transmit as compared to temperature sensor [5]. This type of slot allocation to emergency and nonemergency patient traffic is the responsibility of a BANC. Handling such type of situations $[27,28]$ is the challenging research gap in the cross-layer architecture of WBAN. Thus, the cross-layer architecture has classified the responsibilities of a sensor into Manager and Nonmanager methods and centralized and distributed methods $[13,15,29,30]$. These methods are used in TCP/IP protocol suites to allocate slots as presented in the next section.

\section{Classification of Cross-Layer Designs in Wireless Communication}

TCP/IP protocol suite comprises five layers and was implemented in sensor nodes and across the network. Now, the questions are as follows: (A) how can a sensor internally utilize the functionalities of five layers of TCP/IP for information sharing? and (B) how can a sensor use five layers across the network for information distribution? For answering question (A), two methods are used, namely, Nonmanager and Manager methods [13, 15, 29, 30]. In a similar way, for answering question (B), two methods are used, namely, centralized and distributed methods $[13,15]$. These methods are presented in the following subsections.

3.1. Nonmanager Method. The Nonmanager method is also known as intralayer method which shows the generalized structure of a sensor in the cross-layer architecture where the layers directly share information with each other [14].

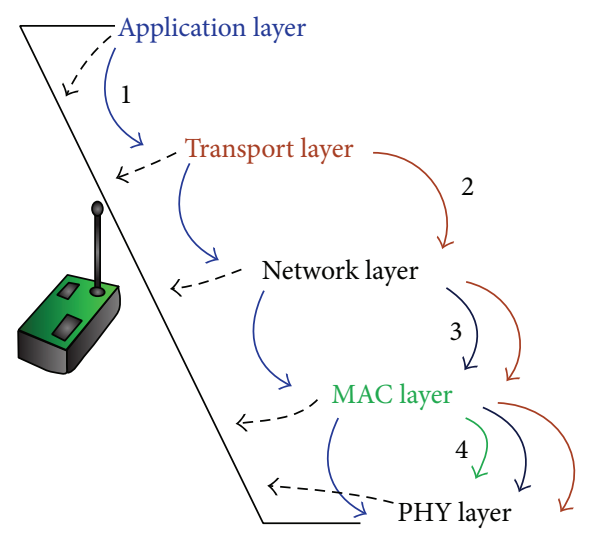

FIGURE 2: Nonmanager method for information sharing (redrawn from $[14,15])$.

Further, this method depicts that application layer communicates sequentially with other four layers of TCP/IP model for information sharing as shown in Figure 2 [14, 15]. In a similar way, the transport layer also shares information with network, MAC, and PHY layers [31]. This process is continued until transmitting data. The information sharing means what functions are needed for transmitting emergency data in each layer of TCP/IP protocol suite on the prioritybasis.

The top-down processes are generated by the transmitter node for transmitting data while bottom-up processes are generated when a sensor receives data from other sensor nodes $[14,15]$. Therefore, the sensor receives data from PHY layer and moves it up to the application layer.

3.2. Manager Method. The Manager method is also known as interlayer method and proposes a vertical plane (VP) for resource sharing among layers [15]. Each layer is not able to communicate directly with other layers but each layer posts its services (functions) in the VP where a specific 


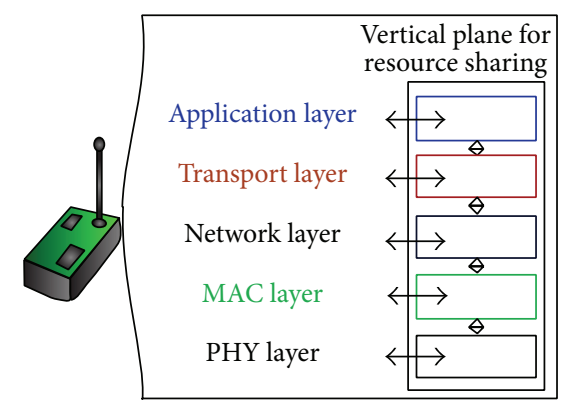

FIGURE 3: Manager method information sharing (redrawn from [14, 15]).

service is assured to be used for specific objective as shown in Figure 3 (redrawn from $[14,15]$ ). For instance, a message needs encryption, data format, compression algorithm, Internet Protocol version 4/6 (IPv4/6) addresses, routing table, MAC and PHY layers services can be ensured during data transmission and reception [32] with the support of a VP. The cross-layer does not change the structure of the TCP/IP protocol stack, but it balances the services in a dynamic way without increasing the overheads for a sensor node [15].

\subsection{Comparison of the Nonmanager and Manager Methods.} Each layer handles the control information, data reception, and transmission directly from other layers in the Nonmanager method [31, 33]. In a similar way, the Manager method also handles data and control information with the support of a VP [14, 15]. As depicted in Figure 2, the application layer shares information regarding the services required for a specific message (emergency and nonemergency data) and each layer visits other layers for information and resource sharing. Further, the application layer services go to the transport layer where the transport layer adds more functionalities to that received message from the application layer. In such way, this process is continued until reaching the PHY layer and transmits the information to the recipient node. In the Manager method, each layer does not visit and share resources (functionalities), but each layer provides the required functionalities for the patient data in the core entity known as vertical plane (VP). The VP operates and provides all types of services from the layers during data transmission and reception. The aim of both methods is not to modify the structure of a sensor node in the cross-layer architecture but to reduce the computational power, storage overhead, and energy consumption of sensors [15].

3.4. Centralized Method. The centralized method comprises multilevels hierarchy for sharing information and resources among sensor nodes in the network as depicted in Figure 4(a) (redrawn from $[14,15]$ ). Level 0 usually contains a centralized node which is known as Base station. The centralized node is responsible for slots (channels) allocation to the rest of levels (lower levels) of sensor nodes in the real time without delay as seen in levels 1 and 2. The purpose of classification of multilevels is to equally distribute the resources and assignments to sensor nodes. For instance, [34] shows that the downlink is proposed between various levels in the crosslayer for allocating channels to sensors in the preallocated time or contention-based sensors. A CDMA-based centralized scheduling access is proposed for real-time video [35]. The classification of the sensor nodes into different levels is based on their desired objectives.

3.5. Distributed Method. In the distributed method, each node forwards data to the destination node directly or via the use of multihops as depicted in Figure 4(b) (redrawn from $[14,15])$. However, it has been noticed that distributed method degrades the network performance in terms of high delay if two nodes select the same node for data transmission and reception that drops data due to congestion.

\section{Cross-Layer Designs for Wireless Body Area Network}

The aim of the cross-layer architecture is to focus on various types of challenging problems and follow them in the routing, MAC, and physical layers of WBAN [4]. Each layer has its own configuration steps for solving the specific problem. For instance, a message needs encryption and data format, so the application layer provides such types of functionalities. This paper presents a generalized overview of the cross-layer in WBAN as shown in Figure 5 where the patient body is covered with different BMSs and they are connected to the BANC in the star topology [36]. BMSs monitor various vital signs of the patient's body that are heartbeat, respiratory rate, blood pressure, temperature, glucose level, EEG, ECG, and EMG $[19,20]$ depending on the patient's disorders. The findings of monitored vital signs are forwarded to the BANC or BAN [8] and BANC forwards these vital signs to the medical staff for necessary action via Base station. The transmission of vital signs on the priority-basis needs routing, MAC, and PHY layers services between BMSs and BANC. Hence, this paper classifies the cross-layer designs into three layers that are discussed in the following subsections.

4.1. Network Layer. Figure 5 shows different BMSs that are connected with the BANC under the criteria when the sensor is under the coverage area of a BANC and there is no traffic transmission; it can use a single-hop [36]. Moreover, if a sensor is away from the coverage area of the BANC and/or sensor contains a minimum energy for transmitting data, then it must use multihops. The multihops based sensors consume minimum energy as compared to the single-hop based ones. For data transmission, the sensor uses Carrier Sense Multiple Access/Collision Avoidance (CSMA/CA) and Time Division Multiple Access (TDMA) [27]. With CSMA/CA based data transmission, each sensor performs contention for channel access with the support of Request-to-Send (RTS), Clear-To-Send (CTS), and Clear Channel Assessment (CCA). With TDMA data transmission, the BANC divides the whole channel into fixed periods of time and assigns the channels to the sensors for transmitting their data in the specified time. The functions of CSMA/CA and TDMA [21] are compared and depicted in Table 2. 


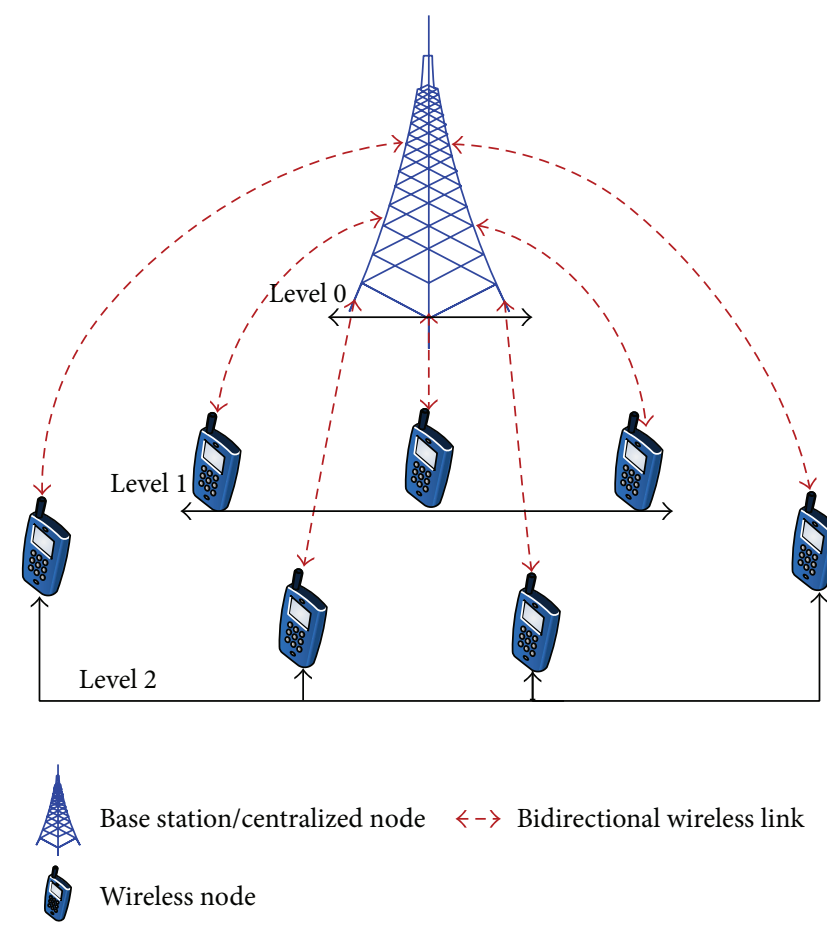

(a)

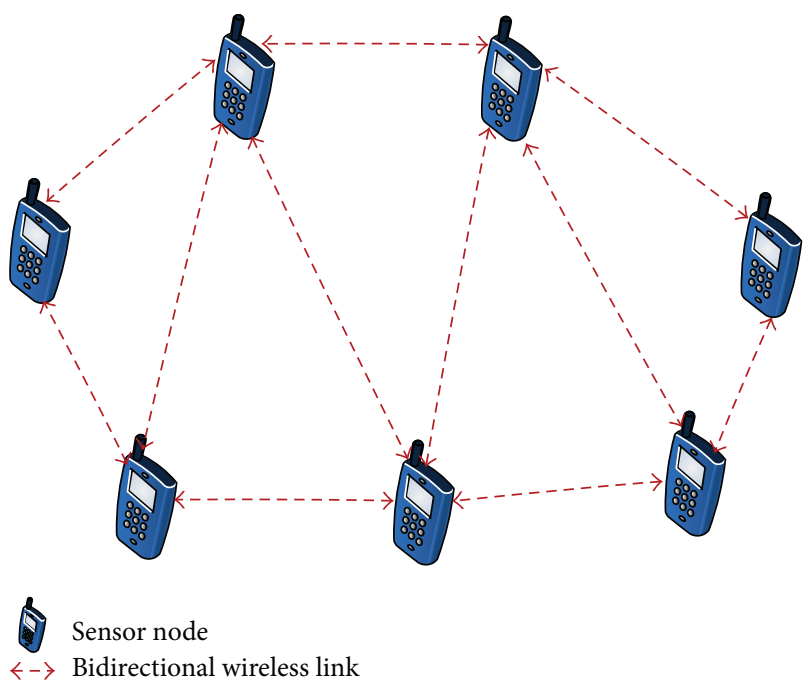

(b)

FIGURE 4: Information and resource sharing in the organization of networks for cross-layer. (a) Centralized method and (b) distributed method. (a) Centralized method is used to share information between sensor nodes in the network while (b) each sensor node used single/more than one path to transmit data to the destination.

TABLE 2: Function comparison of CSMA/CA and TDMA [21].

\begin{tabular}{lcc}
\hline Function & CSMA/CA & TDMA \\
\hline Power consumption & High & Low \\
Bandwidth utilization & Low & Maximum \\
Preferred traffic level & Low & High \\
Dynamic network & Good & Average \\
Effect of packet failure & Low & Latency \\
Synchronization & N/A & Essential \\
\hline
\end{tabular}

The contention-based sensors consume high amount of energy of sensors as compared to TDMA that consumes minimum energy. The judgment is that the TDMA access scheme allocates predefined timeslots to sensors where sensors transmit their data in them. However, the contention minimizes the network performance in terms of high energy consumption, high delay, data collision, and low data reliability and is not suitable on the priority-basis transmitting the emergency data [27]. The Packet Delivery Ratio of TDMA-based data transmission is high as compared to the CSMA/CA due to contention. The drawback of TDMA access scheme is the synchronization before data transmission where CSMA/CA does not need such type of service [21].

4.1.1. Categorization of Patient Data. In literature, the patient data are categorized into CP, RP, DP, and OP [10, 37, 38]. The $\mathrm{CP}$ and $\mathrm{RP}$ are emergency data whereas DP and OP are nonemergency data. The $\mathrm{CP}$ contains low threshold values of vital signs such as low heartbeat and low respiratory rate, while RP contains high threshold values of vital signs such as high heartbeat and high respiratory rate. The OP and DP contain temperature reading, glucose level, and so forth of the patient body. It has been noticed in WBAN that all four types of patient data perform contention to access channel and they degrade the network performance in terms of higher data collision and high delay with low data reliability, and sensors consume high amount of energy [27]. The emergency based BMSs should allocate dedicated slots without contention in order to reduce the addressed issues which are the challenging problem for the patient data in WBAN [39].

4.1.2. Temperature-Aware Based Path Selection. Each BMS keeps updated information of routing table about neighbor BMSs that includes number of hops-count to the destination node, available energy, and temperature-rise of the intermediate BMSs. Before data transmission, the sender BMS verifies the temperature-rise, number of hops, and energy level of each BMS of the whole path. If the temperature of the intermediate BMS is higher than specified threshold values, then that particular BMS (the whole path) will not use this intermediated path for data transmission. The high temperature-rise damages the tissues and skin of the body during monitoring and transmission of findings of vital signs [40]. The reasons are high radio frequency (RF), biosensors 


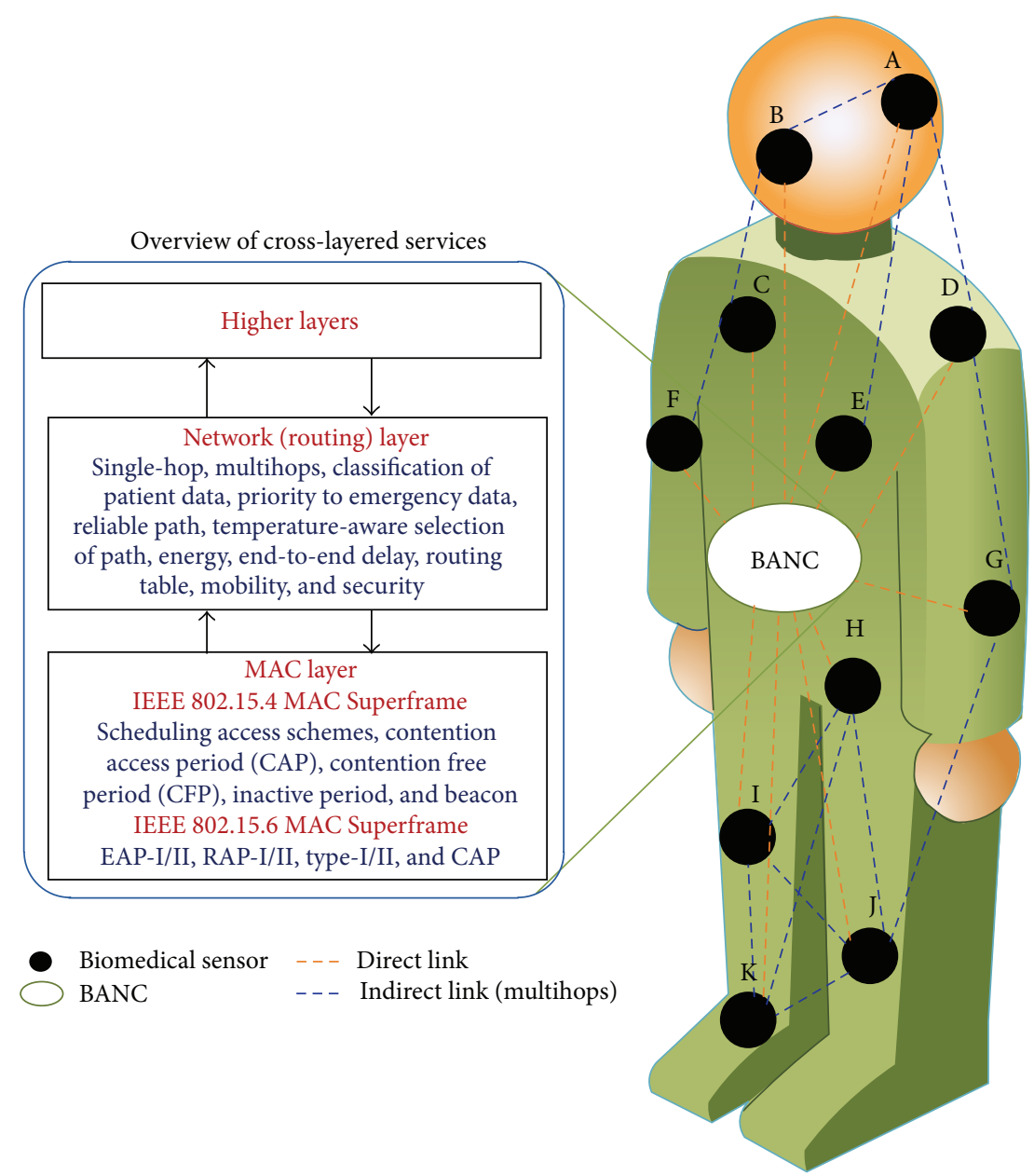

FIgURE 5: Cross-layers architecture in WBAN.

antenna, and sensor circuitry which generate high heat during monitoring and transmission of vital signs. Therefore, [40] used Specific Absorption Rate (SAR) for measuring temperature before the selection of a path for transmitting data as shown in the following:

$$
\mathrm{SAR}=\frac{\sigma|E|^{2}}{\rho}(\mathrm{W} / \mathrm{kg}),
$$

where $\sigma$ is used to transmit electrical heat. $\rho$ is used to find the density level of tissues and $E$ is used to measure the radiation value in the electric field in the patient body.

If the temperature level of a BMS is less than specified threshold values and energy is not enough for transmitting data, in this situation, the transmitter BMS updates the routing table and selects another sensor for path selection [41]. Examples in the cross-layers are DMQoS [10], framework of QoS-aware routing protocol $[42,43]$. However, this process needs higher time for selecting the path which is not acceptable for life threatening vital signs and BMSs high amount of energy.
4.2. MAC Layer. The MAC layer plays a significant role in reducing energy consumption of BMSs. For this purpose, the MAC layer uses reduced duty cycle which reduces the energy consumption of BMSs during contention to access channel [44]. IEEE 802.11, IEEE 802.15, and IEEE 802.15.1 families [45] are not capable of monitoring various vital signs of the patient's body. Therefore, IEEE 802.15.4 MAC Superframe structure is used for monitoring of various vital signs with the capabilities [46] and recently it is known as IEEE 802.15.4 WBAN. Moreover, this paper presents MAC Superframe structures of IEEE 802.15.4 and IEEE 802.15.6 with limitations in the following subsections.

4.2.1. IEEE 802.15.4 MAC Superframe Structure. IEEE 802.15.4 MAC Superframe structure comprises beacon, contention access period (CAP), contention free period (CFP), and inactive period (IP) as shown in Figure 6 (redrawn from [46]). All BMSs are implanted inside the patient body or attached to the surface of a patient body as depicted in Figure 5 under the supervision of a BANC. IEEE 802.15.4 MAC classifies the patient data into normal, periodic, 


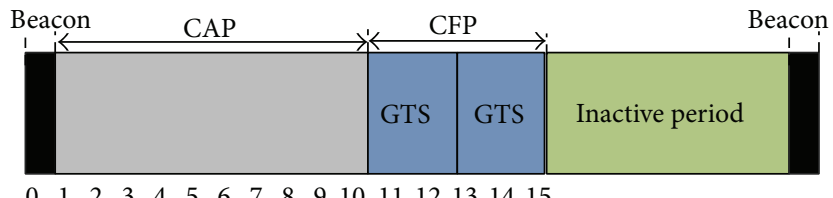

FIGURE 6: 802.15.4 MAC Superframe structure (redrawn from [46]).

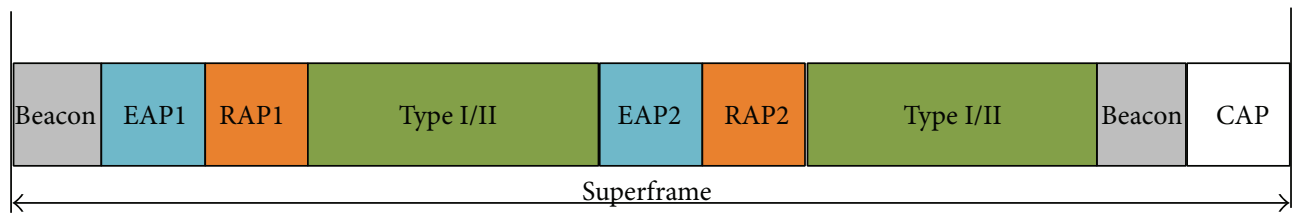

FIgURE 7: 802.15.6 MAC Superframe (redrawn from [3]).

and emergency data and these data in WBAN are known as nature of patient data [46]. The normal data contains temperature reading of the patient body. The periodic data contains blood pressure and glucose whereas emergency data contains low or high threshold values of vital signs.

BANC broadcasts a beacon to all BMSs in the network which includes information of the BANC energy and synchronization. All BMSs perform contention to access channel in the CAP period. The CAP period uses CSMA/CA access scheme and each BMS performs RTS, CTS, and CCA before data transmission. The BANC allocates the CFP slots to those BMSs which have obtained channel access in the CAP period. The CFP slots are based on TDMA access scheme and these slots are Guaranteed Time Slots (GTSs) for transmitting patient data. The IP is used for saving energy when there is no activity being performed. The drawback of IEEE 802.15.4 MAC Superframe structure is that it cannot be used for emergency data where all types of BMSs perform contentions to access channel and does not differentiate between emergency and nonemergency data. Moreover, each sensor uses RTS, CTS, and CCA to access limited 16 slots which degrades the network performance in terms of high delay during contention to access channel, high data collision, and high energy consumption. Due to these shortcomings, numerous research contributions have been made to address the challenging problems and modified IEEE 802.15.4 MAC Superframe structure such as R-MAC [47], HEH-BMAC [48, 49].

4.2.2. IEEE 802.15.6 MAC Superframe Structure. The working group of IEEE 802.15 was decided in 2006 to design a low power sensor for monitoring vital signs of a patient and sportsman during their activities with minimum energy consumption. For this purpose, IEEE 802.15 made a group, known as Task Group 6 (TG6), where they publicized the first draft in 2012 with information of designing of MAC and PHY layers with their frame structure [3].

Figure 7 shows IEEE 802.15.6 MAC Superframe structure that contains beacon, exclusive access phase (EAP-I/II), random access phase (RAP-I/II), type-I/II, and contention access period $(C A P)[3,50]$. The BANC broadcasts a beacon message in the network for synchronizing the clocks with all BMSs. The CSMA/CA and slot Aloha access schemes are used in IEEE 802.15.6 MAC. EAP-I and EAP-II are used for carrying emergency data whereas $R A P-I, R A P-I I$, and $C A P$ are used for carrying normal data. Type-I indicates emergency data while type-II indicates normal data.

IEEE 802.15.6 MAC is the same drawback of high energy consumption of BMSs during contention to access channel, which affects the performance in terms of low data reliability with high delay and high energy consumption. Further, there are no such rules defined which can assist emergency based BMSs for allocating dedicated slots.

4.2.3. Comparison of MACs of IEEE 802.15.4 and IEEE 802.15.6. IEEE 802.15.4 Wireless Sensor Network (WSN) and IEEE 802.15.6 Wireless Body Area Network (WBAN) are capabilities of sensing/monitoring and detecting of the event from the desired environment in advance and inform the authorities about the outcomes. Therefore, this study compares IEEE 802.15.4 MAC and IEEE 802.15.6 MAC with various parameters [22] as depicted in Table 3. WSN is used to detect an event from environment like detection of mines from battlefield, pipeline leakage, and temperature of a room, whereas WBAN is used to monitor various vital signs of a patient and sportsman [20]. The coverage area of WSN's sensors is 100 meters as compared to WBAN's BMSs area which is $2-5$ meters coverage area. WSN supports 65,000 sensors in the network while WBAN supports 256 BMSs. The limitation of WSN sensors consumes maximum energy as WBAN BMS consumes minimum energy which has been observed during monitoring of vital signs periodically in the sleep mode. However, the duty cycles of a sensor have reduced the energy consumption [27]. The propagation medium of WSN is air while WBAN uses a human body, that is, tissues and skin. WSN sensors do not use SAR [40] as it does not require the outside environment (e.g., battlefield) while the biomedical sensors use SAR [40] to measure temperature level of biomedical sensors before transmission of the monitored data to the destination node. Both IEEE 802.15.4 and IEEE 802.15.6 MACs are almost the same access schemes such as CSMA/CA, TDMA, and Slotted Aloha for channel 
TABLE 3: MAC functionalities comparison of IEEE 802.15.4 and IEEEE 802.15.6 [22].

\begin{tabular}{|c|c|c|}
\hline Objectives & MAC 802.15 .4 & MAC 802.15.6 \\
\hline Environment & $\begin{array}{l}\text { Sensors monitor various environments like home } \\
\text { appliances, automatic operations, and battlefield but } \\
\text { can be used for health domain as well }\end{array}$ & $\begin{array}{l}\text { Sensors monitor patient body for the health } \\
\text { condition, sports, and game }\end{array}$ \\
\hline Network deployment range & 10 to 100 meters & 2 to 5 meters \\
\hline Network coverage & Large & Medium \\
\hline $\begin{array}{l}\text { Min-to-max sensors } \\
\text { support }\end{array}$ & 10 to 65000 & 3 to 256 \\
\hline Energy consumption & $20 \mathrm{~mW}$ to $35 \mathrm{~mW}$ & $0.01 \mathrm{~mW}$ to $40 \mathrm{~mW}$ \\
\hline Frequency band & ISM & $\begin{array}{l}\text { Approved by medical authorities for } \\
\text { in/on-body }\end{array}$ \\
\hline Data transmission medium & Air & Air, on-body, and in-body \\
\hline Data transmission rate & $20 \mathrm{~Kb} / \mathrm{sec}$ to max. $250 \mathrm{~Kb} / \mathrm{sec}$ & $50 \mathrm{~Kb} / \mathrm{sec}$ to $10 \mathrm{Mb} / \mathrm{sec}$ \\
\hline $\begin{array}{l}\text { Safety for in/out organs of } \\
\text { human body }\end{array}$ & No, but can use the specific absorption rate (SAR) & Yes, use of the SAR \\
\hline Access scheme & CSMA/CA, TDMA, FDMA, and Aloha & CSMA/CA, TDMA, and Aloha \\
\hline Controls overhead & Low & High \\
\hline
\end{tabular}

\begin{tabular}{|c|c|c|c|c|c|}
\hline \multicolumn{2}{|c|}{ Synchronization header (SHR) } & $\begin{array}{c}\text { Physical header } \\
\text { (PHR) }\end{array}$ & \multicolumn{3}{|c|}{ Physical payload (PH load) } \\
\hline
\end{tabular}

FIGURE 8: IEEE 802.15.4 physical frame structure (redrawn from [52]).

contention and data transmission. The control overheads in the WSN are low as compared to WBAN which has high overheads. The reason is that WSN is used for homogeneous nature of data while WBAN is used for heterogeneous nature of patient data. The discussion ends with the fact that IEEE 802.15.4 MAC provides all types of the required functionalities in health domain which will be provided by underlying IEEE 802.15.6 MAC WBAN. For instance, [51] improves MAC IEEE 802.15.4 Superframe structure for minimum energy consumption, and [49] focuses on the patient's emergency data.

4.3. Physical Layer. The physical layer provides the functionalities to activate and deactivate the radio signal of a sensor for transmitting data, efficient signals modulation, and controlling of frame and data on the sender and reception. This paper presents physical frame structure of IEEE 802.15.4 [52] and IEEE 802.15.6 [3] in the following subsections.

4.3.1. IEEE 802.15.4 Physical Layer Frame Structure. IEEE 802.15.4 physical frame structure comprises $S H R, P H R$, and PH load as depicted in Figure 8 (redrawn from [52]). The SHR consists of "preamble sequence" and "start of frame delimiter." The PHR contains "frame length" whereas PH load contains "frame control," "data sequence number," and "frame check sequence."

The preamble sequence occupies 4 bytes and are used when a BANC broadcasts a message of synchronization association with sensors in the network. The start of frame delimiter shows the start of the frame to the receiver node on the receiving side and occupies 1 byte in 802.15.4 PHY layer header. The frame length indicates the total length of a frame and occupies 1 byte. Each sensor transmits a channel request to the BANC and BANC replies back with updated acknowledgment whether the channel status is under congestion or free of congestion. This process is verified in the frame control header of the physical layer which reserves 2 bytes. Further, more than one sensor shares one channel with using DeMUX-ID and transmits data accordingly. Therefore, the data sequence number counts the number of the received frames and differentiates among other sensors data. It occupies 1 byte in the physical frame structure. The frame check sequence (FCS) maintains the integrity of frames during transmission and it uses 2 bytes. The sender sensor generates a hash value of the intended frames, attaches the generated hash value to the message, and transmits to the receiver sensor. On the receiver side, the receiver sensor also generates the hash value of the received message and compares it to the received hash value message. If the hash values of both messages are matched, then the receiver accepts the message; otherwise, it drops the received message as the message integrity has been violated.

4.3.2. IEEE 802.15.6 Physical Layer. The responsibility of IEEE 802.15.6 physical layer is to activate and deactivate radio signals of antenna and use the CCA before channel reservation [3]. Therefore, IEEE 802.15.6 classifies the physical 
layer into Narrow Band (NB), Human Body Communication (HBC), and Ultra Wide Band (UWB) [3] for transmitting patient data. The NB physical layer is the prime choice for transmitting data for the long distance communication while UWB is the second choice for the short range communication. This paper presents three types of data transmission in the following subsections.

(a) IEEE 802.15.6 NB Physical Layer. IEEE 802.15.6 NB PHY provides functionalities of data transmission/reception, listens to the channel, and performs CCA before data transmission, activation, and deactivation of the radio signals. The NB PHY comprises physical layer convergence procedure (PLCP) preamble, PLCP header, and physical layer service data unit (PSDU) [3]. The PCLP preamble assists in recovering the contents of a damaged message and the packet detection and provides synchronization at the receiver side. The PCLP header uses different modulation and demodulation techniques for signals that are Differential 8Phase-Shift Keying (D8PSK), Differential Binary Phase-Shift Keying (DBPSK), and Differential Quadrature Phase-Shift Keying (DQPSK) [3].

(b) IEEE 802.15.6 HBC Physical Layer. The HBC PHY layer uses Electrostatic Field Communication (EFC) for data transmission. The packet structure comprises PLCP preamble, Start Frame Delimiter (SFD), PLCP header, and PSDU [3]. Further, the HBC uses a gold-code generator and generates a 64-bit code for PLCP preamble and SFD. The preamble has used a 64 bit code four times for synchronization. The SFD informs the receiver about new frame and detects the starting point of a new frame. The preamble header is transmitted with the support of Frequency Shift Code (FSC). The PCLP header also contains Pilot information and Cyclic Redundancy Check-8 (CRC-8). The Pilot information has the same functionality as SFD and inserts bits in the frame for synchronization between the transmitter and receiver sensors. The CRC-8 is used to detect an error in the received frames at the receiver side.

(c) IEEE 802.15.6 UWB Physical Layer. The UWB is used for short range communication and has been designed for onbody sensors. The design of a UWB physical layer is very low complexity, high performance, and low power consumption as compared to the NB and HBC [3]. The frame structure of the UWB comprises synchronization header (SHR), PHY header (PHR), and PSDU [3]. Moreover, the SHR consists of two subfields that are preamble and SFD. The PHR encrypts the transmitter message before transmission and transports the whole messages in the PSDU towards the receiver node.

Table 4 shows various modulation techniques that are used in the UWB PHY: frequency modulation (FM) UWB and Impulse Radio (IR) UWB [23]. The UWB PHY uses 11 channels and these channels are integrated into low band and high band [23]. The low band uses three channels ( 0 to 2 ) and the high band uses eight channels (3-10). The bandwidth of each channel is $499.2 \mathrm{MHz}$. The low bandwidth frequencies are $3494.4 \mathrm{MHz}, 3993.6 \mathrm{MHz}$, and $4492.8 \mathrm{MHz}$, while high bandwidth frequencies are $6489.6 \mathrm{MHz}, 6988.8 \mathrm{MHz}$,
TABLE 4: UWB physical layer modulation techniques [23].

\begin{tabular}{|c|c|c|c|c|}
\hline $\begin{array}{l}\text { Method of } \\
\text { operation }\end{array}$ & PHY & $\begin{array}{c}\text { Data rate } \\
\text { (kbps) }\end{array}$ & Modulation & $\begin{array}{c}\text { Operation } \\
\text { band }\end{array}$ \\
\hline \multirow{2}{*}{ Default } & IR-UWB & 487.5 & On-off signaling & $\begin{array}{l}\text { Low and } \\
\text { high bands }\end{array}$ \\
\hline & FM-UWB & 250 & $\begin{array}{l}\text { CP-BFSK, } \\
\text { FM-UWB }\end{array}$ & High band \\
\hline QoS & IR-UWB & 487.5 & DPSK & $\begin{array}{c}\text { Low and } \\
\text { high bands }\end{array}$ \\
\hline
\end{tabular}

7488.0 MHz, $\quad$ 7987.2 MHz, $\quad$ 8486.4 MHz, $\quad$ 8985.6 MHz, 9484.8 MHz, and 9984.0 MHz [23].

\section{Cross-Layered Protocols}

The cross-layer protocols provide multilevel functionalities across routing layer, MAC layer, and PHY layer in WBAN [53]. The aim of cross-layer is to draw attentions towards the challenging problems in all three layers at a time, for example, how to assign on the priority-basis a high reliable path to emergency data in routing layer and how to assign the slot in the MAC protocol for such type of problem. Hence, the following are objectives of the cross-layer protocols:

(i) Selection of the shortest and reliable path for the successful delivery of patient data to the destination node.

(ii) Selection of path based on node's temperature and the residential energy.

(iii) Updating the routing table of the whole network regarding paths detail, energy level, and temperature of the nodes.

(iv) Load-aware data transmission in the network.

(v) Classification of nature of patient data according to the objectives.

(vi) Slots allocations to nodes in MAC layer on the emergency basis.

(vii) Utilization of the contentions and preallocated slots to the nodes in convenient way.

(viii) Nodes away from the coordinator that must be aware of this type of situation and use the relay nodes for data transmission between the transmitter node and coordinator.

(ix) The nodes that should act as relay nodes between sender and receiver nodes when a signal becomes weak.

(x) The Narrow Band (NB) and Ultra Wide Band (UWB) that reduce the signal fading and researchers ought to use them for patient's data transmission.

(xi) Use of reduced duty cycles for reducing energy consumption, queuing process, priority-based slots allocation, and temperature based path selection. 
These functionalities are provided in three layers of WBAN. Moreover, this paper presents various research contributions which have been made for the cross-layers in the following.

The WASP (wireless autonomous spanning tree protocol) is the cross-layer protocol and uses MAC and routing layers to achieve multihop-counts communication with minimum energy consumption as compared to single-hop communication [54]. The proposed protocol [54] is used for intrabody communication and comprises 6 steps as shown in the following:

(1) Assign addresses to nodes in the network.

(2) Each child is under the supervision of a sink and follows the WASP scheme with a data transmission slot.

(3) The child receives data in a silent period from its lower tree children nodes.

(4) The child forwards the received data to the parent node and the parent node transmits the data to the destination node.

(5) New node uses contention for a channel in requesting JOIN-REQUEST message.

(6) Acknowledgement is used to verify data transmission and comprises ACK-0, which means data not received, and ACK-1, which means data received.

The sink/parent node divides the time slots into fixed number of slots in order to transmit and receive data. During data transmission, the parent nodes listen to their children conversation in the tree and similarly the children also listen to their parents conversation [54]. The new node joins the parent network with a JOIN-REQUEST message. The parent node does not reply to JOIN-REQUEST message of a new node if the parent node is busy with other nodes for their data transmission. The purpose of this ignorance of a message is not to degrade the performance of the network in terms of high delay, low data reliability, and high energy consumption of the nodes. When all data transmission is finished, then a new node can join the network. However, there is no mechanism available in this scheme for avoiding the collision of packets. If the parent/children does not reply in time " $n$ ", the parent/children considers that the parent/node has left the network. The node address consists of 6 bits which are not enough for large and scalable network. If a node receives the damaged packet from the transmitter node, then there is a limitation in this protocol that is how to inform the transmitter node about the damage packets in order to retransmit it which has not been considered in this WASP scheme [54].

The cascading information retrieval by controlling access with distributed slot assignment (CICADA) [55] protocol proposes and has the same frame format as presented in IEEE 802.15.4 MAC. The proposed protocol uses treelike topology and every child node in the tree acts like a parent node of its subchildren nodes where this process depends on the density level of tree. Further, the proposed protocol divides the slots into different slots and uses two subcycles that are "control subcycles" and "data subcycles." These two subcycles are used before allocation of time slots to subparent nodes and their children. The control subcycle scheme comes from the root parent node to their children and the data subcycles scheme comes from children to their parent nodes. The purpose of the control subcycle is to allow child nodes to transmit their control scheme data and deactivate the signals after data transmission. The data subcycles contains total length, depth of the tree, and data period. The total length is used for all nodes where they send their schemes for slot reservation while the tree depth is used for the length of tree. The data period is used to receive data from their children to its parent while the waiting period is used to switch off the radio signal when a node is in the waiting state. If a new node wants to join the network, it must send the "JOIN-REQUEST" message to the parent node. However, if the new node does not send the data packet/hello packet, then the control packet of the parent node performs two or more consecutive cycles to verify that particular node availability. After these consecutive cycles, the parent node considers that a new node has been disjoined or misplaced from the network. The same case also happens with the parent node. If the parent node does not reply in 2 or more consecutive cycles, it is considered that a parent node has left the network and one of their children must act as a parent node. The benefit of this protocol is being an energy efficient approach with distributive manner resource sharing in the whole tree.

The Time Zone Coordinated Sleep Scheduling (TICOSS) protocol is proposed in [56] for multihop communication. The V-Table is proposed in this protocol which maintains information of the sender and receiver data, switch-off of the radio signal when a node is not transmitting data, assignment of addresses to nodes, transmission schedule, and division of the whole network into different time zones. All these kinds of information of Table 5 propagate to their children in the tree with the support of a gateway or parent node. Moreover, the personal area network coordinator (PANC) is used as gateway to collect the data from full functional device (FFD) and reduced functional device (FFDs) which have been used in the network. IEEE 802.15.4 MAC has the following limitations [56] as presented:

(a) Design for single-hop communication with the concept of a single parent node and children.

(b) No scalability supports for large networks with the combination of more than one star topology.

(c) Cluster based star topology that creates overlapping issues during data transmission which produces a high interference and high packet collision.

(d) Nodes consuming high energy due to contention for slots in the CAP period.

(e) IEEE MAC 802.15.4 that uses 64-bit long address whereas the proposed TICOSS protocol uses 16-bit short addresses.

The proposed protocol [56] follows the MERLIN [57] concept for assigning time zones to the network nodes. Moreover, the proposed protocol provides three unique 


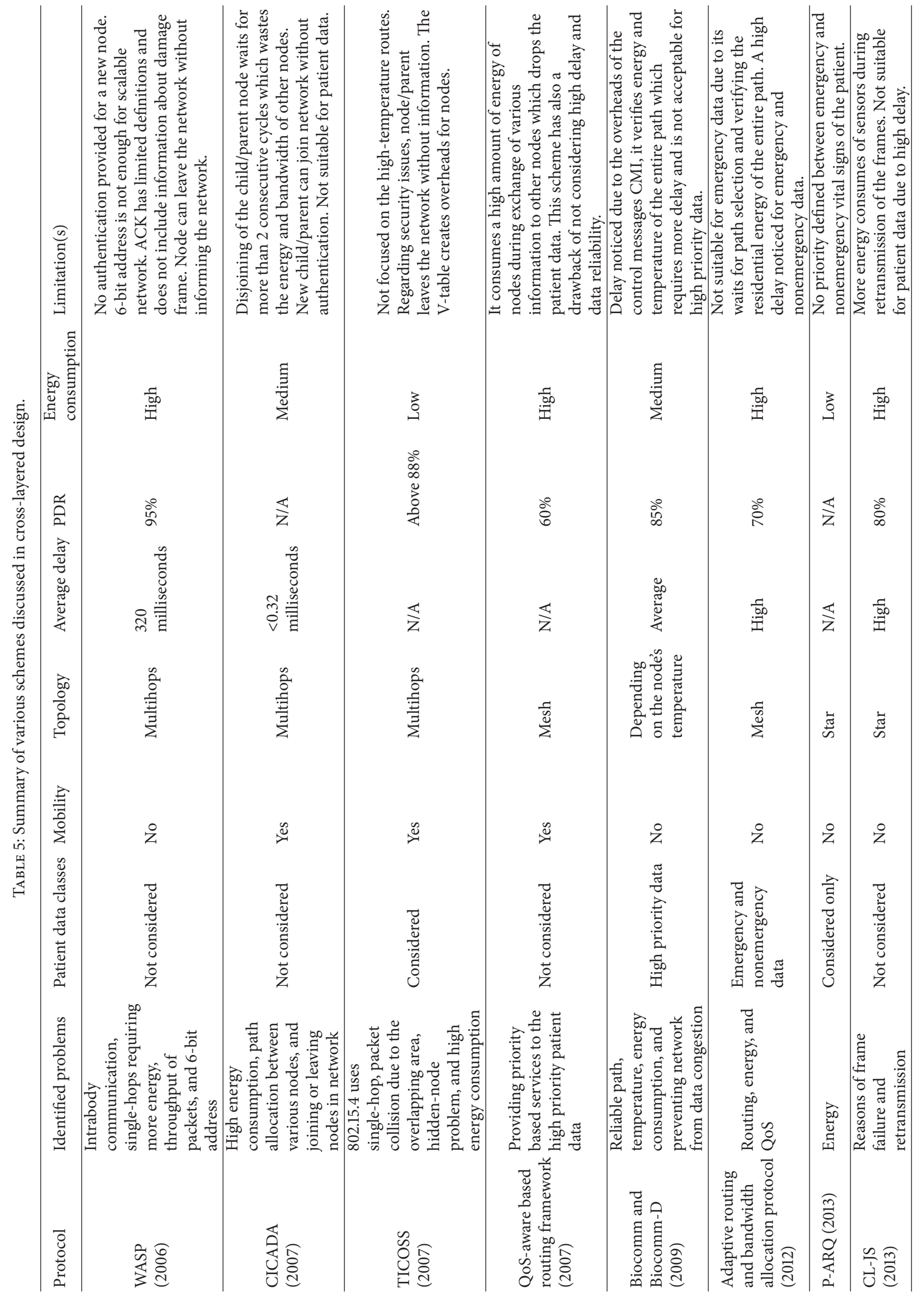




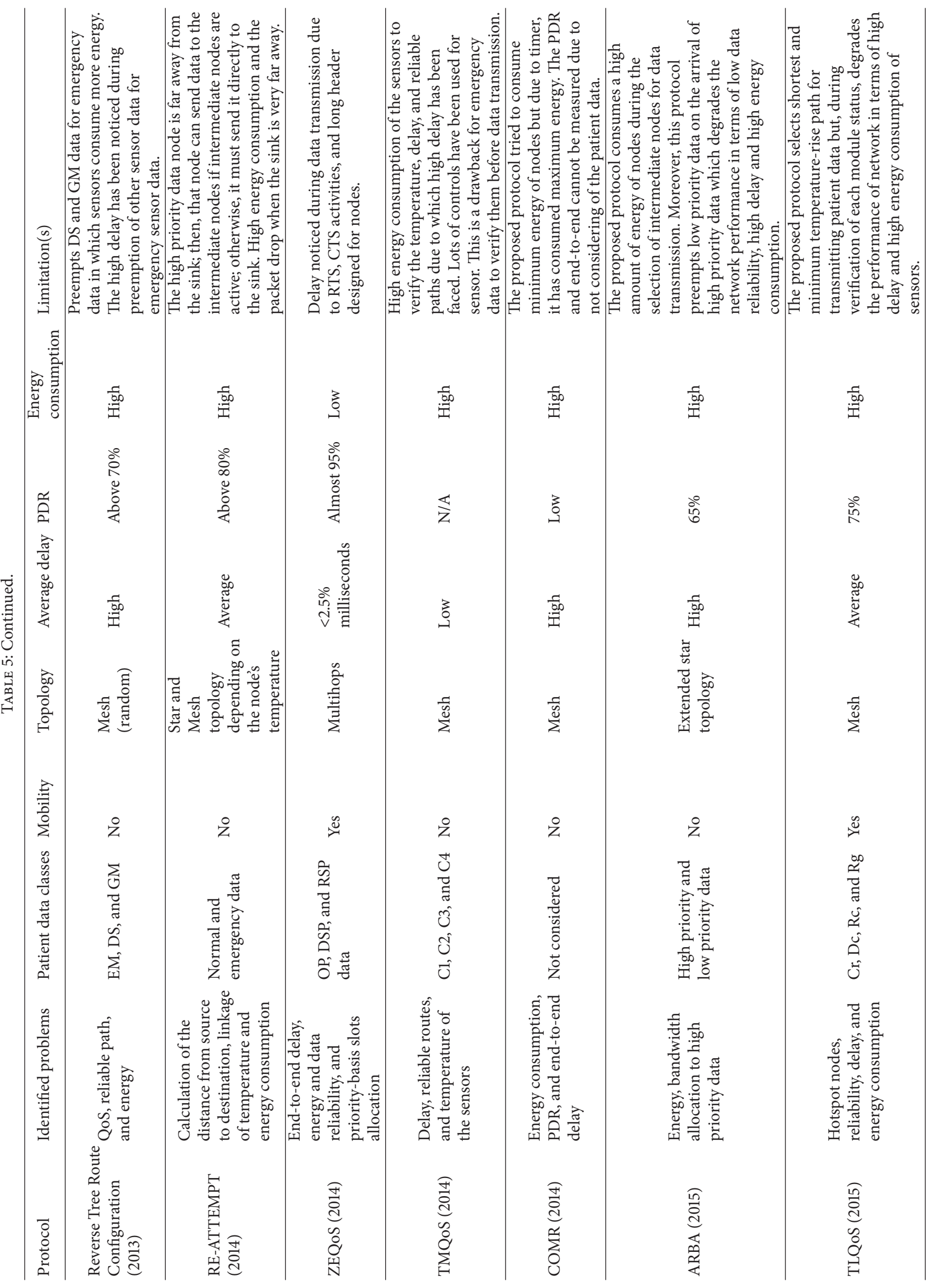


features which makes it feasible to implement sensors inside the patient body as described in the following:

(1) All the nodes must ensure waking up at the correct time for transmitting or receiving data and consume minimum energy.

(2) Detect the packets from the overlapping area due to hidden-node problem.

(3) Select the shortest path among available paths near to the PANC or gateway for the packet transmission.

The upstream, downstream, and local broadcast provide allocating the buffer to the packets on First In First Out (FIFO) policy. In the upstream broadcast, the packets transmit from the closer source to the gateway. In downstream broadcast, the packets come from the lower time zone nodes density to the upper time zone nodes density and further the packets are transmitted to the gateway. The nodes in the local broadcast send or receive information from the network. However, this proposed protocol [56] does not focus on the high-temperature routes and the node/parent leaves the network without informing the particular node. The VTable creates overheads for nodes during selection of paths and data transmission.

QoS-aware based routing framework proposes to focus on the designing of QoS routes, connection setup, and maintenance and guaranteed based delivery of a high priority packet and the willingness of a node to forward the packets as a router [42]. Therefore, the proposed framework comprises an Application Programming Interface (API), QoS metrics, packet queuing and scheduling, and system information repository as shown in Figure 9 (redrawn from [42]). The API interacts with different options of a system for providing ondemand QoS. The QoS metrics provide four types of QoS that are (i) QoSMetricsSet, (ii) SendPacket, (iii) RecPacket, and (iv) admission and service level. Moreover, the QoSMetricsSet provides services to end-to-end packet delivery and consumes the minimum energy of the nodes. The SendPacket contains sender and receiver IDs, data priority schemes, and payload information. The payload data obtains data from the payload packet with the support of a RecPacket (Received packet) function. The admission and service type is the final submodule with aims to inform the application users of the network conditions. The second main module is routing service which establishes and maintains the sessions between users and also informs the sender of the neighbor nodes status. The maintenance of sessions is performed in the packet queuing and scheduling module which divides the packets into different eight priority services. These priority services have been provided by packet payload. The highest priority numbers are 7 and 8 . These two numbers are assigned to the most critical patient packets. The priority services from 1 to 6 are assigned to different packets which can be normal data, on-demand services, and file uploading, and so forth. The system information repository module maintains the link quality of the network and keeps some nodes active for transmitting a high priority data. However, this proposed scheme [42] consumes a high amount of energy of nodes during exchanging of different information and drops other nodes data. Moreover, this proposed scheme is also not considering energy consumption and data reliability which degrades the network performance in terms of high delay during data transmission.

The performance enhancement of all nodes in the network in terms of high data reliability with the shortest and energy efficient path is one of the challenging problems. Therefore, the proposed protocol, Biocomm protocol, proposes to design the cross-layer messaging interface (CMI) [58]. The CMI provides the functionality of connectivity between routing and MAC layers as the intermediate path to exchange information which has been described in the following:

(i) Before data transmission, the CMI indicates the hightemperature nodes and routes (e.g., hotspots).

(ii) CMI minimizes the energy consumption of nodes in terms of turning off their radio signals.

(iii) CMI protects the network from data congestion during data transmission.

The buffer space (BS) memory is not enough in the network layer if huge amount of data comes for storage. Hence, the BS of routing layer forwards the received data to BS of MAC layer and keeps the network away from data congestion, avoids data dropping, and assigns a reliable path to data if it is a high priority data. The MAC layer uses MAC logic and keeps the node alive or in inactive status for consuming minimum energy. Thus, the node status information is forwarded to the network layer with the support of a CMI interface. Before data transmission to the destination node, the sender node verifies the channel availability from the Last-Active Node (LA) message. The Biocomm-D is the extended version of the Biocomm [58] and its aim is to take care of the delay-sensitive medical application data. However, the proposed protocol [58] faces a high delay due to control messages, verification of energy, and temperature of the entire path which is not acceptable for high priority data.

The emergency data is a priority to reallocate the bandwidth as compared to nonemergency data in the network. The allocation of bandwidth to emergency data depends on the residential energy of sensors. Therefore, [59] proposes crosslayer based on an adaptive routing and bandwidth allocation protocol which comprises four phases that are topology discovery, energy-aware routing, bandwidth allocation, and load balancing routing. Each sensor broadcasts a hello message in the neighbor region for discovering sensor nodes in the topology discovery phase and maintains a neighbor table considering information of the connection request, data transmission, and connection termination. The sender sensor selects those sensors which have high residential energy and the shortest hops-count to the coordinator. These two parameters have been used in the path energy. The bandwidth allocation phase allocates slots on the priority-basis to the low/high threshold values (emergency data) first as compared to the temperature sensor data (nonemergency data). In this special case, the emergency data allocates a high bandwidth and terminates the nonemergency data during transmission 


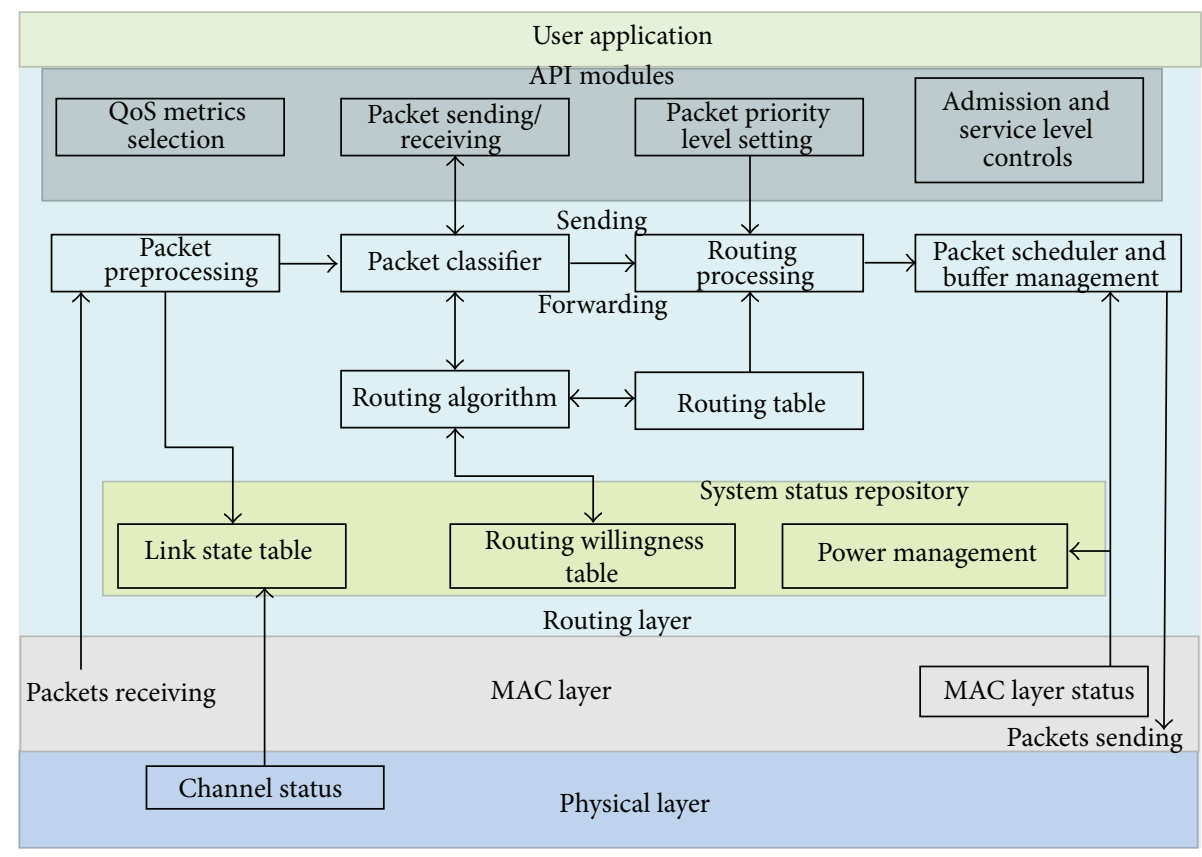

FIGURE 9: Framework of QoS-aware routing protocol (redrawn from [42]).

to the sink. The accepted and nonaccepted messages are used in the load balancing routing phase where sensors can accept the request of other sensors for forwarding data towards the sink. The acceptance and rejection of a request are based on the energy level of the concerned sensor and also depend on the condition of the vital sign as stated in ARBA [60]. However, the energy consumption of this proposed protocol [59] is high in terms of the route selection and maintaining the table which degrades the performance of the network in terms of high delay with low data reliability.

The wireless radio module is the most energy consumption component in the wireless sensor. The MAC layer can reduce the energy consumption of sensors using the reduced duty cycle. Therefore, the cross-layer based on this study [61] proposes Controllable Phase Locked Loop (CPLL) and CPLL automatic repeat request (P-ARQ) method. The CPLL adds a Frequency Compensation (FC) module which can reduce the state transition from the sleep state to $\mathrm{Tx} / \mathrm{Rx}$ state. The FC module is connected with the Voltage Controller Oscillator (VCO) module for setting up frequency of a sensor. Moreover, the P-ARQ [61] uses three-way handshakes to establish the connection between a slave sensor and master sensor in the network initialization phase. The MAC header uses PPL Lock and PPL Count to lock the connection and count the number of transmitted/received frames. The energy consumption of sensors has been minimized in the proposed methods due to TDMA access scheme. However, this scheme has not investigated delay, Packet Delivery Ratio, and priority-based slots allocation to emergency and nonemergency data.

The frame retransmission occurs due to three types of problems that are as follows: (i) if more than one sensor transmits its data at the same time to the same receiver, (ii) Hidden Device Problem (HDP), For example, sensor " $C$ " receives data from sensors " $A$ " and " $B$ " while both sensors $\mathrm{A}$ and $\mathrm{B}$ are unaware of each other during data transmission, and (iii) the channel fading problem when receiver receives data which is not acceptable for the receiver [44]. Due to these shortcomings, [44] proposes an algorithm which detects an error of the transmitted frame at PHY layer of IEEE 802.15.4 and informs sender about the error. The sender follows the procedure of retransmission of a frame from the MAC layer. Further, the proposed algorithm is the cross-layer judgment scheme (CL-JS) [44] and it uses the Energy Detection (ED) and Average Count Number (ACN) parameters that justify the error reason and retransmission of the damaged frames. Both parameters are used at the receiver side. The ED examines the channel (signal) strength and variation in the channel while the ACN tries to correct the errors in the received frames. Moreover, the proposed algorithm uses four steps for investigating the errors in the frames [44] as follows:

(1) If $\left(\mathrm{ED} \geq \mathrm{ED}_{\text {thresd_values }}\right) \& \&\left(\mathrm{CAN}<\mathrm{ACN}_{\text {thresd_values }}\right)$, then the frame is accepted and the sender transmits it in the CAP of IEEE 802.15.4 MAC.

(2) If $\left(\mathrm{ED}<\mathrm{ED}_{\text {thresd_values }}\right) \& \&\left(\mathrm{CAN}<\mathrm{ACN}_{\text {thresd_values }}\right)$, then the frame is accepted and the sender transmits it in the CAP of IEEE 802.15.4 MAC.

(3) If ( $\left.\mathrm{ED}<\mathrm{ED}_{\text {thresd_values }}\right) \& \&\left(\mathrm{CAN} \geq \mathrm{ACN}_{\text {thresd_values }}\right)$, then frame transmission failed due to signal fading and the sender retransmits the frame in the CFP of IEEE802.15.4 MAC.

(4) If ( $\left.\mathrm{ED} \geq \mathrm{ED}_{\text {thresd_values }}\right) \& \&\left(\mathrm{CAN} \geq \mathrm{ACN}_{\text {thresd_values }}\right)$, then frame transmission failed due to collision/HDP and the sender retransmits the frame in the CFP of IEEE802.15.4 MAC. 
However, the proposed algorithm [44] degrades the performance of the network in terms of high energy consumption of sensors to retransmit the frame which is not acceptable for emergency data.

The challenging problem is how to define the shortest path for those sensors that are away from the coordinator. The work in [4] proposes an algorithm which is known as "Reverse Tree Route Configuration." At the beginning of communication, the coordinator broadcasts a beacon message to all sensors in the network. The corner sensors in the network accept only the beacon message from the nearest sensors and make an association with them for data transmission and reception. Through this process, the whole network becomes convergent. The patient data are classified into emergency data (EM), delay-sensitive (DS) data, and General Monitoring (GM) data. In the routing layer, the coordinator preempts the DS and GM data for EM data if the coordinator receives it, and if there is no path for data transmission. The EAP-I, EAP-II, and type-I are subfields of IEEE 802.15.6 MAC and are used for emergency data, whereas RAP-I, RAP-II, CAP, and type-II are used for DS and GM patient data. The Remaining Time (RT) scheduler is used for transmitting DS and GM data which have been preempted earlier for EM data. The Packet Delivery Ratio (PDR) of sensors is acceptable in terms of reliable data transmission. However, the preemption of DS and GM data for emergency data consumes a high amount of energy which degrades the network performance in terms of high delay for emergency data.

The improved version of M-ATTEMPT [62] is Reliability Enhanced-Adaptive Threshold Based Thermal Unaware Energy-Efficient Multihop Protocol (RE-ATTEMPT) [63]. The proposed protocol examines the path loss and energy consumption. The proposed protocol uses four phases of the routing protocol to reduce the deficiencies of M-ATTEMPT protocol. The first phase is initialization phase where all nodes broadcast a "hello" message for exchanging information with all neighbor nodes in the network along with a sink. The purpose of this message is to identify the shortest routes to the destination for high priority data packets during transmission. The second phase is the routing phase where emergency data (high priority packet) is transmitted directly to the sink or uses the shortest hop-count paths. For instance, those nodes can transmit emergency and normal data to the sink directly which are under the direct coverage area of the sink. Those nodes are away from the sink node and they want to transmit emergency data. The option for this type of transmission is that they must transmit data through intermediate nodes to the sink if the intermediate nodes are active. Otherwise, they must transmit emergency data to the sink directly which consumes high amount of energy. Moreover, the normal data is transmitted by the same aforementioned method. The third phase is scheduling phase and it contains TDMA access scheme. This scheme is used for normal data transmission while the emergency based sensors perform contention in the contention free period (CFP) of MAC layer for transmitting data. The final phase is data transmission phase which is used for allocating slots to nodes and preventing data collision between nodes. However, the proposed protocol consumes a high energy and drops data if the sink is away and sensor contains minimum energy for transmitting data. Another limitation of this scheme is that it does not consider low/high threshold values of vital signs.

Energy and QoS-aware based ZEQoS [43] routing protocol is proposed and compared with LOCALMORE [64] and DMQoS [10] in terms of categorization of patient data, reliable data transmission, and energy consumption. The energy consumption of the proposed protocol is high but as compared with EPR, QPRD and QPRR protocols are very low. Moreover, the patient data has been categorized in this protocol into Ordinary Packets (OPs), Delay-Sensitive Packets (DSPs), and Reliability Sensitive Packets (RSPs). The proposed protocol calculates communication costs, end-toend delay, and reliability from source to destination node before data transmission. These parameters help in increasing throughput of the network. The proposed framework comprises MAC layer and routing layer modules [43]. The module of MAC layer contains MAC Receiver, Reliability Module, Delay Module, and MAC Transmitter while network layer module contains packet classifier, hello packet, and routing service module. The MAC Receiver module receives "hello" or patient data and forwards it to the concerned module of the network layer. The Reliability Module updates the counter of number of transmitted packets and acknowledges the sender node and destination node. The Delay Module of the MAC layer assigns a channel bandwidth to the patient data. The MAC Transmitter receives the patient packet and hello packet. Moreover, the MAC transmitter forwards this type of data to the MAC layer and the transmission policy used FIFO with the support of CSMA/CA. All these kinds of information come from the network layer. The network layer is classified into various services that are packet classifier, routing services module (RSM), hello protocol module, and QoS-aware queuing module (QQM) [43]. The packet classifier receives a hello packet and patient data from the MAC Receiver and forwards them to the concerned service module. If the packet contains a hello message, then it is forwarded to the hello packet module; otherwise, it is forwarded to the routing service module. The routing service module classifies patient data into four classes as aforementioned and updates the routing tables of the neighbor nodes for selecting an efficient-QoS path. The hello protocol module helps in creating the neighbor tables and broadcasts a hello message in the network. The last module is $Q Q M$ and this module knows the first priority should be given to DSP, second priority is to RSP, and the third priority is to OP for allocating slots. However, the proposed protocol [43] uses RTS and CTS control messages before data transmission which degrades the network performance in terms of a high delay with low data reliability and the long header for nodes during data transmission.

The cross-layer based thermal-aware multiconstrained intrabody QoS (TMQoS) routing protocol is proposed [65] for reducing delay in order to achieve high data reliability during data transmission. These problems occur due to high temperature-rise which degrades the network performance. The proposed protocol [65] develops routing architecture and it interacts with the routing layer and MAC layer as shown in Figure 10 (redrawn from [65]). The biomedical sensors 


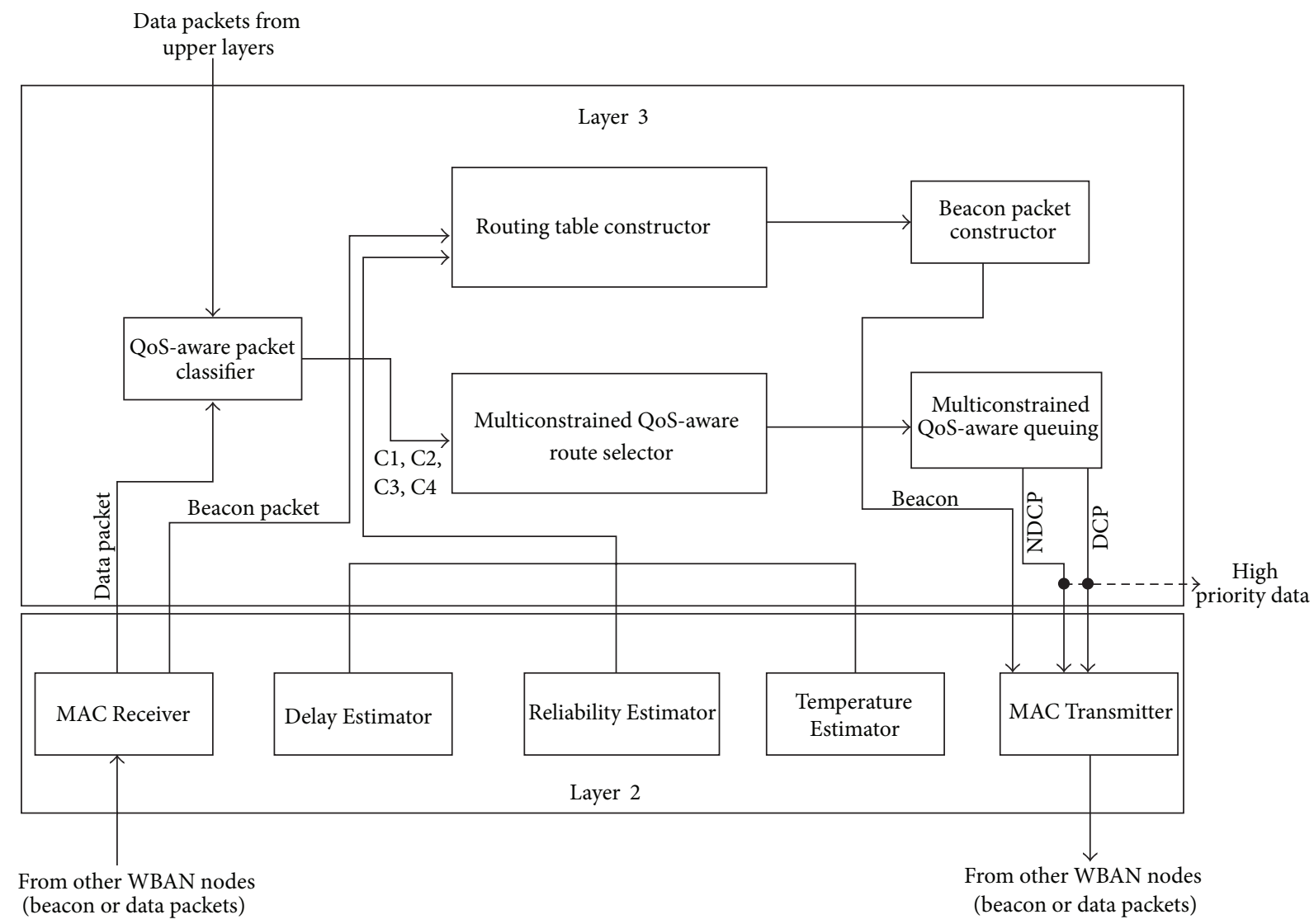

FIGURE 10: TMQoS routing architecture (redrawn from [65]).

are implanted inside the patient's body and connected with a body coordinator (BC) through multiple paths. As depicted in Figure 10, the MAC Receiver module receives a beacon message from neighbor sensors and forwards it to the routing table constructor of each sensor. The routing table constructor contains information about delay, reliable path, hop-counts to BC, and temperature-rise of intermediate sensors. The Delay Estimator module measures delay from the intermediate sensors to a destination BC. The Reliability Estimator module measures QoS of the link while the Temperature Estimator module measures the temperature-rise of sensors. These three modules update the routing table of a sensor and broadcast the beacon table constructor information to all neighbor sensors through the MAC Transmitter module. In this stage, the sensor is familiar to delay, reliable, and temperature-rise of the intermediate sensors and selects the most optimal routes. Moreover, the QoS-aware packet classifier module receives vital signs data from upper layers or from the MAC Transmitter. This module classifies the patient data into $\mathrm{C} 1, \mathrm{C} 2, \mathrm{C} 3$, and $\mathrm{C} 4$. The $\mathrm{C} 1$ data is the highest priority data and cannot delay it such as EEG, EMG, and heart beat. The $\mathrm{C} 2$ data is the second priority data which may contain respiratory rate reading of a patient. The C3 data is the third priority data and contains video/audio streaming whereas $\mathrm{C} 4$ is the fourth priority data and contains temperature reading. The QoS-aware packet classifier module forwards these four types of patient data to the multiconstrained QoS-aware route selector module where this module selects the most appropriate routes as aforementioned in three modules. At that moment, the selector module transmits data to multiconstrained QoS-aware queuing and the queuing module maintains the Delay Constrained Packets (DCPs) queue for $\mathrm{C} 1$ and $\mathrm{C} 3$ patient data, respectively, while Nondelay Constrained Packets (NDCPs) queue are maintained for C2 and C4 patient data, respectively. Finally, data is transmitted through MAC Transmitter module. However, the proposed routing architecture [65] consumes high energy of sensors during verification of temperature-rise and selection of a reliable path for data transmission. These factors increase a high delay and they have the drawback for emergency data to verify each time of these elements before data transmission which is not acceptable for real-time data.

The Cross-Layer Opportunistic MAC/Routing (COMR) protocol proposes to reduce energy consumption during data transmission of nodes and increase Packet Delivery Ratio (PDR) with minimum end-to-end delay [66]. Therefore, the proposed protocol uses relay nodes for transmitting the packets with the support of a timer. The timer is based on the received signal strength indicator (RSSI) and residential energy of the selected nodes as relay nodes. For data transmission, the proposed protocol introduces four types of handshaking mechanism that are distributed interframe spacing (DIFS), RTS, CTS, and acknowledgement (ACK). The sender node cannot compete for a channel and must wait for DIFS before transmitting the packets if the channel is idle. On the successful completion of this process, the sender 
node competes for channel and performs many backoffs if the channel is busy. During this channel competition, the sender node transmits the RTS packet and waits for a timer. The timer calculates the contention period, waiting period of a sender node for the RTS and CTS messages, and the propagation delay of the RTS and CTS messages. However, the energy consumption of this proposed protocol [66] is high if the size of payload is increased; for example, the long generated report of an ECG requires a high amount of time during transmission. The PDR of this proposed protocol is lower due to timer based selection of relay nodes which consumes high amount of energy. These elements degrade the network performance in terms of low data reliability and high delay which is not suitable for patient data.

An adaptive routing and bandwidth allocation protocol (ARBA) [26] proposes to investigate the problems of energy consumption and allocation of bandwidth to the sensory data. The proposed protocol comprises four phases that are topology discovery, energy-aware routing tree construction, rate and bandwidth allocation, and load balancing routing and energy usage [26]. In topology discovery phase, each node exchanges a hello message with other nodes in the network for creating the registry. The registry keeps the information of the nodes for establishing the connection in terms of the connection request, data transmission, and connection termination. In the energy-aware routing tree construction, a high residential energy node selects an intermediate node (relay) for transmitting of the source node data to BAN. The priority of allocation of high rate bandwidth to the high priority data is performed in the rate and bandwidth allocation phase. Further, this phase terminates the allocation of high rate bandwidth from the low priority data if the high priority data need a large amount of bandwidth. The final phase is load balancing routing and energy usage that selects the node based on its importance of data and high residential energy. However, the proposed protocol [26] consumes a high amount of energy of nodes during selection of intermediate nodes for data transmission and discovery of the entire topology. Moreover, this protocol terminates data transmission of low priority data on the arrival of high priority data. Another drawback of this protocol is not focusing on changing the structure of the topology which is not acceptable for high priority data in terms of low data reliability, high delay, and high energy consumption of the intermediate nodes.

There are three types of challenging problems that occurred during data transmission from the source node to the destination node inside the patient body that are hotspot nodes problems during sensing of a patient vital signs, transmission of sensory data to the BAN which heats up the surroundings of the tissues and skin of the body, and high energy consumption. These problems degrade the network performance in terms of low data reliability with high delay and throughput. To overcome these problems, [67] proposes a thermal-aware QoS routing protocol (TLQoS) for layer 2 and layer 3 modular architecture. The proposed protocol classifies the patient data into critical (Cr) traffic, delay constrained (Dc) traffic, reliability constrained (Rc) traffic, and regular (Rg) traffic. The Cr sensory data can be a lower delay and higher reliability such as ECG, EEG. The Dc sensory data can be loss for a short period of time without delay such as audio/video based patient data. The Rc sensory data need higher reliability without delay such as respiratory rate while $\mathrm{Rg}$ sensory data does not constrain delay and reliability such as temperature and blood pressure. Moreover, layer 3 comprises Delay Module, Reliability Module, Temperature Module, and Queuing Manager. In a similar way, layer 2 comprises MAC Receiver, Delay Estimator, Reliability Estimator, Temperature Estimator, and MAC Transmitter. The Delay Module is used for transmitting Cr and Dc traffic. Both types of traffic depend on the number of hops if the number of hops exceeds the particular hops. This Delay Module also protects patient traffic from looping. The Reliability Module is used for $\mathrm{Rc}$ and $\mathrm{Cr}$ traffic. This module protects both types of patient traffic from a large number of hops-counts, hotspot routes, and swapping of front sensor data with back sensor data. The Temperature Module follows the same rules of Delay Module and Reliability Module and is used to carry $\mathrm{Rg}$ traffic. The Temperature Module selects those nodes for data transmission that has minimum temperature-rise and minimum hops-count. The Queuing Manager is the fourth module of layer 3 and it classifies the buffer into DCR and RQ. The DCR buffer occupies Cr and Dc traffic whereas RQ buffer occupies Rc and Rg traffic. The first module of layer 2 is Delay Estimator and it selects delay-sensitive routes for $\mathrm{Cr}$ and $\mathrm{Dc}$ traffic. The Reliability Estimator tries to lose the minimum packet and it uses window mean with EWMA approach for calculation of reliability. The Temperature Estimator uses Specific Absorption Rate (SAR) [68] for measuring radiation level of tissues of the implanted sensors. The MAC Receiver and QoS-aware packet classifier make differences between a hello packet and data packet. The hello packet informs BAN about network status while the data packet contains the patient traffic. The proposed protocol is rich of modules providing different services to forward patient data. However, each module in this proposed protocol creates a high delay during verification of the conditions of module and consumes high amount of energy of the sensors which degrades the performance of network in terms of high delay and high energy consumption.

The aim of cross-layer protocol is to design specific task for routing, MAC, and PHY layers such as achieving high efficiency of the network in terms of minimizing the energy consumption and delay, achieving high data reliability, and allocating the first slot to emergency data as compared to nonemergency data. Hence, this paper presents various research contributions which have been made for design of cross-layer protocols. Table 5 presents the summaries of existing protocols in terms of mobility, topology, delay, PDR, and energy consumption in the comparative study.

\section{Comparative Study}

This paper compares existing cross-layer protocols in terms of classification of patient data, mobility, topology, average delay, Packet Delivery Ratio (PDR), and energy consumption as shown in Table 5. The WASP [54] scheme uses fixed 
time slots to transmit data, but the energy consumption is high due to unlimited resources provided to on-demand nodes. Moreover, [54] does not support end-to-end delivery of packets. It does not provide acknowledgment of a damaged frame to the sender node but it allows a new node to join the network which is not available in the existing crosslayer protocols. However, the limitation is that a node can leave the network anytime without informing the network. CICADA [55] is the enhanced type of WASP [54] and it uses "waiting period" to keep off radio signal when a node is in waiting state. Hence, the energy consumption of [55] is average (medium) as compared to [54]. Both schemes [54, 55] have the same problems when a parent/children node imparts the network without informing the responsible node. IEEE 802.15.4 MAC has many challenging problems as discussed in TICOSS [56]. It follows time slot scheduling concept of MERLIN [57] in which all nodes must ensure waking up in the correct time for sending/receiving data and saves energy. The work in [56] provides better results in the energy consumption and packet delivery delay (PDR) as compared to [55]. The energy consumption of this scheme [42] is the main drawback and degrades the network performance in terms of higher delay and lower data reliability. The proposed architecture of Biocomm [58] is for routing and MAC layer where both layers communicate with each other through CMI. The work in [58] keeps data away from the congested nodes, avoids dropping of data, and assigns a reliable path to data if the data is of higher priority. Further, [58] indicates the hotspot nodes and link before data transmission as compared to [54-56]. Comparing the Biocomm [58] to [54], both protocols consume minimum energy. The Biocomm-D is the extended version of [58] which provides acceptable results in terms of energy consumption and identification of hotspot nodes before data transmission. The work in [59] consumes higher amount of energy of sensors in selection of paths. It finds the sensors with higher residential energy. The higher energy consumption in path selection degrades the network performance in terms of higher delay in case of emergency data as experienced in $[44,58]$.

The energy consumption of [59] is higher as compared to $[55,56,58]$. However, [59] considers emergency and nonemergency data of a patient which have not been considered in [54-56]. The PDR of [59] is lower as compared to [54, 56, 58]. The P-ARQ [61] does not consider the patient data but the energy consumption of this protocol [61] is lower as compared to $[54,59]$ and is outperformed as shown in [56]. The CL-JS [44] provides data reliability in terms of retransmission of frames. However, this process degrades the performance of network in terms of delay and higher energy consumption of sensors as compared to $[55,56$, 59]. The PDR of [44] is better than [59] but is not good enough compared to $[54,56,58]$. The work in [4] is more suitable for emergency data where this proposed protocol preempts the nonemergency data from allocated slots and allocates slots to emergency data on arrival for transmitting first as compared to all protocols in this paper. However, the higher energy consumption and higher delay have been noticed due to preemption process of nonemergency data as compared to $[55,58]$. The RE-ATTEMPT [63] is the extended version of M-ATTEMPT [62] scheme. The scheme in [63] examines path loss and energy consumption. The work in [63] divides packets into normal and emergency packet as compared to ZEQoS [43] which classifies patient data into three categories as shown in Table 5. The energy consumption of RE-ATTEMPT [63] is better than M-ATTEMPT [62] but is not better than ZEQoS [43]. The energy consumption of ZEQoS [43] scheme is higher as compared with EPR, QPRD, and QPRR schemes. The work in [43] has the same framework structure as LOCALMORE [64] and DMQoS [10]. The energy consumption of [65] is higher as experienced in $[4,43,44,54,59]$. However, $[43,55,56,58,61]$ are having lower energy consumption as compared to [65]. The work in [65] classifies patient data into four classes also as discussed in [43]. Further, [65] uses temperature-aware approach for selecting the nonhotspot paths from source to destination as $[58,63]$ use the same approaches for path selection. The drawback of this proposed protocol [66] is higher energy consumption during selection of relay nodes for transmitting data. Moreover, the PDR and end-to-end delay have not been improved due to long waiting on the timer based for channel contention and data transmission. The works in $[43,59,63,65]$ classify the patient data into different classes. The mobility options are used in $[43,55,56]$. The STAR and MESH topology are used in all schemes. The works in $[56,61]$ schemes do not target average delay. In the similar way, [55, $61,65]$ also do not examine PDR. The energy consumption of these schemes $[43,56,61]$ is low, whereas $[55,58]$ have medium energy consumption and $[4,44,54,59,63,65]$ are experiencing higher energy consumption. These analyses are shown to address the aforementioned problems with new prototypes.

The energy consumption of [26] is higher due to preemption of low priority data on arrival of high priority data, changing the structure of topology, and selection of intermediate nodes for data transmission. These parameters degrade the network performance in terms of lower data reliability and higher delay. The proposed protocol [67] consumes major amount of energy of sensors during verification of status of each module which degrades the network performance because of higher delay and lower data reliability.

We can reduce energy consumption and delay and enhance data reliability and PDR with new approaches. New algorithms and protocols, for routing and MAC layers in WBAN, are required for addressing the aforementioned challenges in existing protocols.

\section{Conclusion}

The design of new routing protocols, MAC Superframe structures, and PHY frames are the challenging problems in the health applications of WBAN. The aforementioned problems draw attention to design optimized solutions in the cross-layer architecture and protocols. Therefore, the routing layer and both MAC and PHY (physical) layers of IEEE 802.15.4 and IEEE 802.15.6 structures have been investigated for the cross-layer in WBAN. The routing layer has been 
investigated in terms of selection of single and multihops path, energy-aware and temperature-aware selection of paths, and classification of patient data into different priority levels; emergency data is the first priority to transmit on the reliable path, mobility, and topology. IEEE 802.15.4 MAC and IEEE 802.15.6 MAC Superframe structures have been compared and it was found that most of the research studies used IEEE 802.15.4 MAC Superframe structure for WBAN due to its easy manipulation for patient traffic and implementation. In a similar way, IEEE 802.15.4 and IEEE 802.15.6 PHYs frame structures have been compared. IEEE 802.15.6 PHY frame provides $\mathrm{NB}, \mathrm{HBC}$, and UWB approaches for transmitting data.

All functionalities that will be provided by IEEE 802.15.6 for MAC and PHY layers in WBAN have been already provided by IEEE 802.15.4 for MAC and PHY layers in WBAN as described in Table 3 and investigated in Section 5.

The researchers ought to design an optimized model for routing, MAC, and PHY layers. The proposed model should be capable of selecting an efficient path for emergency based BMSs in terms of low temperature and high residential energy and minimum hop-counts. For MAC layer, there should be a dynamic solution for emergency based BMSs so that they do not need to perform contention and dedicated slots should be provided without interrupting contention of nonemergency based BMSs. PHY layer should provide an efficient modulation technique which facilitates minimum time and energy consumption for changing analog signal into a digitized pattern.

\section{Conflict of Interests}

The authors declare that there is no conflict of interests regarding the publication of this paper.

\section{Acknowledgment}

This research work is supported by Universiti Teknologi Malaysia (UTM), under Grant no. Q.J130000.2528.06H00.

\section{References}

[1] G. Acampora, D. J. Cook, P. Rashidi, and A. V. Vasilakos, "A survey on ambient intelligence in health care," Proceedings of the IEEE, vol. 101, no. 12, pp. 2470-2494, 2013.

[2] G. Fortino, D. Parisi, V. Pirrone, and G. Di Fatta, "BodyCloud: a SaaS approach for community Body Sensor Networks," Future Generation Computer Systems, vol. 35, pp. 62-79, 2014.

[3] S. Movassaghi, M. Abolhasan, J. Lipman, D. Smith, and A. Jamalipour, "Wireless body area networks: a survey," IEEE Communications Surveys \& Tutorials, vol. 16, no. 3, pp. 16581686, 2014.

[4] H. Ben Elhadj, S. Boudjit, and L. C. Fourati, "A cross-layer based data dissemination algorithm for IEEE 802.15.6 WBANs," in Proceedings of the International Conference on Smart Communications in Network Technologies (SaCoNeT '13), pp. 1-6, IEEE, Paris, France, June 2013.

[5] F. Ullah, A. Khelil, A. A. Sheikh, E. Felemban, and H. M. A. Bojan, "Towards automated self-tagging in emergency health cases," in Proceedings of the IEEE 15th International Conference on e-Health Networking, Applications and Services (Healthcom '13), pp. 658-663, IEEE, Lisbon, Portugal, October 2013.

[6] A. Boulis, D. Smith, D. Miniutti, L. Libman, and Y. Tselishchev, "Challenges in body area networks for healthcare: the MAC," IEEE Communications Magazine, vol. 50, no. 5, pp. 100-106, 2012.

[7] S. X. S. Xiao, A. Dhamdhere, V. Sivaraman, and A. Burdett, "Transmission power control in body area sensor networks for healthcare monitoring," IEEE Journal on Selected Areas in Communications, vol. 27, no. 1, pp. 37-48, 2009.

[8] E. Jovanov and A. Milenkovic, "Body area networks for ubiquitous healthcare applications: opportunities and challenges," Journal of Medical Systems, vol. 35, no. 5, pp. 1245-1254, 2011.

[9] S. González-Valenzuela, X. Liang, H. Cao, M. Chen, and V. C. M. Leung, "Body Area Networks Sergio," August 2012, pp. 1738.

[10] M. A. Razzaque, C. S. Hong, and S. Lee, "Data-centric multiobjective QoS-aware routing protocol for body sensor networks," Sensors, vol. 11, no. 1, pp. 917-937, 2011.

[11] N. Ababneh, N. Timmons, and J. Morrison, "Cross-layer optimization protocol for guaranteed data streaming over Wireless Body Area Networks," in Proceedings of the 8th IEEE International Wireless Communications and Mobile Computing Conference (IWCMC '12), pp. 118-123, Limassol, Cyprus, August 2012.

[12] O. Rajput, S. Qureshi, A. R. Solangi, Z. Aziz, and F. K. Shaikh, "Applicable operational mechanisms to assist visually impaired people-a WSN perspective," in Proceedings of the 5th International Conference on Information and Communication Technology for the Muslim World (ICT4M '13), pp. 1-6, IEEE, Rabat, Morocco, March 2013.

[13] N. A. F. Foukalas, V. Gazis, and N. Alonistioti, "Cross-layer design proposals for wireless mobile networks: a survey and taxonomy," IEEE Communications Surveys and Tutorials, vol. 10, no. 1, pp. 70-85, 2008.

[14] B. Fu, Y. Xiao, H. J. Deng, and H. Zeng, "A survey of cross-layer designs in wireless networks," IEEE Communications Surveys and Tutorials, vol. 16, no. 1, pp. 110-126, 2014.

[15] H. Zeng, K. Joon, J. Deng, B. Fu, Y. Xiao, and J. Jeski, "Proactive and adaptive reconfiguration for reliable communication in tactical networks," in Defense Transformation and Net-Centric Systems, vol. 8405 of Proceedings of SPIE, pp. 1-9, 2012.

[16] I.-H. Chuang, C.-T. Hsieh, and Y.-H. Kuo, "An adaptive crosslayer design approach for network security management," in Proceedings of the 13th International Conference on Advanced Communication Technology (ICACT '11), pp. 1085-1089, IEEE, Seoul, Republic of Korea, February 2011.

[17] L. D. P. Mendes and J. J. P. C. Rodrigues, "A survey on cross-layer solutions for wireless sensor networks," Journal of Network and Computer Applications, vol. 34, no. 2, pp. 523-534, 2011.

[18] J. Wang and G. L. Smith, "A cross-layer authentication design for secure video transportation in wireless sensor network," International Journal of Security and Networks, vol. 5, no. 1, pp. 63-76, 2010.

[19] M. Chen, S. Gonzalez, A. Vasilakos, H. Cao, and V. C. M. Leung, "Body area networks: a survey," Mobile Networks and Applications, vol. 16, no. 2, pp. 171-193, 2011.

[20] X. Lai, Q. Liu, X. Wei, W. Wang, G. Zhou, and G. Han, "A survey of body sensor networks," Sensors, vol. 13, no. 5, pp. 5406-5447, 2013. 
[21] S. Marinkovic, C. Spagnol, and E. Popovici, "Energy-efficient TDMA-based MAC protocol for wireless body area networks," in Proceedings of the 3rd International Conference on Sensor Technologies and Applications (SENSORCOMM '09), pp. 604609, Athens, Greece, June 2009.

[22] N. Thanh, A. Vernhet, and Z. Rosenzweig, "Gold nanoparticles in bioanalytical assays and sensors," in Frontiers in Chemical Sensors, Springer Series on Chemical Sensors and Biosensors, pp. 261-277, Springer, Berlin, Germany, 2005.

[23] M. Li and M. Zhuang, "An overview of physical layers on wireless body area network," in Proceedings of the International Conference on Anti-Counterfeiting, Security and Identification (ASID '12), pp. 1-5, IEEE, Taipei, Taiwan, August 2012.

[24] B. Latré, B. Braem, I. Moerman, C. Blondia, and P. Demeester, "A survey on wireless body area networks," Wireless Networks, vol. 17, no. 1, pp. 1-18, 2011.

[25] E. G. Spanakis, V. Sakkalis, K. Marias, and A. Traganitis, "Cross layer interference management in wireless biomedical networks," Entropy, vol. 16, no. 4, pp. 2085-2104, 2014.

[26] N. Ababneh, N. Timmons, and J. Morrison, "A cross-layer QoSaware optimization protocol for guaranteed data streaming over wireless body area networks," Telecommunication Systems, vol. 58, no. 2, pp. 179-191, 2015.

[27] Z. A. Khan, M. B. Rasheed, N. Javaid, and B. Robertson, "Effect of packet inter-arrival time on the energy consumption of beacon enabled MAC protocol for body area networks," Procedia Computer Science, vol. 32, pp. 579-586, 2014.

[28] A. Pantelopoulos and N. G. Bourbakis, "A survey on wearable sensor-based systems for health monitoring and prognosis," IEEE Transactions on Systems, Man and Cybernetics Part C: Applications and Reviews, vol. 40, no. 1, pp. 1-12, 2010.

[29] V. Srivastava and M. Motani, "Cross-layer design: a survey and the road ahead," IEEE Communications Magazine, vol. 43, no. 12, pp. 112-119, 2005.

[30] G. Carneiro, J. Ruela, and M. Ricardo, "Cross-layer design in 4G wireless terminals," IEEE Wireless Communications, vol. 11, no. 2, pp. 7-13, 2004.

[31] T. Kwon, H. Lee, S. Choi et al., "Design and implementation of a simulator based on a cross-layer protocol between MAC and PHY layers in a WiBro compatible IEEE 802.16e OFDMA system," IEEE Communications Magazine, vol. 43, no. 12, pp. 136-146, 2005.

[32] S. Khan, Y. Peng, E. Steinbach, M. Sgroi, and W. Kellerer, "Application-driven cross-layer optimization for video streaming over wireless networks," IEEE Communications Magazine, vol. 44, no. 1, pp. 122-130, 2006.

[33] C. Luo, F. R. Yu, H. Ji, and V. C. M. Leung, "Cross-layer design for TCP performance improvement in cognitive radio networks," IEEE Transactions on Vehicular Technology, vol. 59, no. 5, pp. 2485-2495, 2010.

[34] R. Ferrús, L. Alonso, A. U. Juliana, X. R. Ballesté, J. PérezRomero, and F. J. Casadevall, "Cross-layer scheduling strategy for UMTS downlink enhancement," IEEE Communications Magazine, vol. 43, no. 6, pp. S24-S28, 2005.

[35] H. Jiang, W. Zhuang, and X. Shen, "Cross-layer design for resource allocation in $3 \mathrm{G}$ wireless networks and beyond," IEEE Communications Magazine, vol. 43, no. 12, pp. 120-126, 2005.

[36] M. M. Alam and E. Ben Hamida, "Surveying wearable human assistive technology for life and safety critical applications: standards, challenges and opportunities," Sensors, vol. 14, no. 5, pp. 9153-9209, 2014.
[37] Z. Khan, N. Aslam, S. Sivakumar, and W. Phillips, "Energyaware peering routing protocol for indoor hospital body area network communication," Procedia Computer Science, vol. 10, pp. 188-196, 2012.

[38] I. Anjum, N. Alam, M. A. Razzaque, M. M. Hassan, and A. Alamri, "Traffic priority and load adaptive MAC protocol for QoS provisioning in body sensor networks," International Journal of Distributed Sensor Networks, vol. 2013, Article ID 205192, 9 pages, 2013.

[39] B. Roman, I. Wassell, and I. Chatzigeorgiou, "Scalable crosslayer wireless access control using Multi-Carrier Burst Contention," IEEE Journal on Selected Areas in Communications, vol. 29, no. 1, pp. 113-128, 2011.

[40] Q. Tang, N. Tummala, S. K. S. Gupta, and L. Schwiebert, "Communication scheduling to minimize thermal effects of implanted biosensor networks in homogeneous tissue," IEEE Transactions on Biomedical Engineering, vol. 52, no. 7, pp. 12851294, 2005.

[41] M. Tabandeh, M. Jahed, F. Ahourai, and S. Moradi, "A thermalaware shortest hop routing algorithm for in vivo biomedical sensor networks," in Proceedings of the 6th International Conference on Information Technology: New Generations (ITNG '09), pp. 1612-1613, IEEE, Las Vegas, Nev, USA, April 2009.

[42] X. Liang and I. Balasingham, "A QoS-aware routing service framework for biomedical sensor networks," in Proceedings of the 4th IEEE International Symposium on Wireless Communication Systems (ISWCS '07), pp. 342-345, IEEE, Trondheim, Norway, October 2007.

[43] Z. A. Khan, S. Sivakumar, W. Phillips, and B. Robertson, "ZEQoS: a new energy and QoS-aware routing protocol for communication of sensor devices in healthcare system," International Journal of Distributed Sensor Networks, vol. 2014, Article ID 627689, 18 pages, 2014.

[44] H.-W. Tseng and Y.-R. Chuang, "A cross-layer judgment scheme for solving retransmission problem in ieee 802.15.4-based wireless body sensor networks," IEEE Sensors Journal, vol. 13, no. 8, pp. 3124-3135, 2013.

[45] R. Cavallari, F. Martelli, R. Rosini, C. Buratti, and R. Verdone, "A survey on wireless body area networks: technologies and design challenges," IEEE Communications Surveys \& Tutorials, vol. 16, no. 3, pp. 1635-1657, 2014.

[46] C. Li, B. Hao, K. Zhang, Y. Liu, and J. Li, "A novel medium access control protocol with low delay and traffic adaptivity for wireless body area networks," Journal of Medical Systems, vol. 35, no. 5, pp. 1265-1275, 2011.

[47] S. Ullah, "RFID-enabled MAC protocol for WBAN," in Proceedings of the IEEE International Conference on Communications (ICC '13), pp. 6030-6034, IEEE, Budapest, Hungary, June 2013.

[48] E. Ibarra, A. Antonopoulos, E. Kartsakli, and C. Verikoukis, "HEH-BMAC: hybrid polling MAC protocol for WBANs operated by human energy harvesting," Telecommunication Systems, vol. 58, no. 2, pp. 111-124, 2015.

[49] J. S. Yoon, G.-S. Ahn, S.-S. Joo, and M. J. Lee, "PNP-MAC: preemptive slot allocation and non-preemptive transmission for providing QoS in body area networks," in Proceedings of the 7th IEEE Consumer Communications and Networking Conference (CCNC '10), pp. 1-5, IEEE, Las Vegas, Nev, USA, January 2010.

[50] S. Ullah, M. Mohaisen, and M. A. Alnuem, "A review of IEEE 802.15.6 MAC, PHY, and security specifications," International Journal of Distributed Sensor Networks, vol. 2013, Article ID 950704, 12 pages, 2013. 
[51] L. Lin, K.-J. Wong, A. Kumar, S. L. Tan, and S. J. Phee, "An energy efficient MAC protocol for mobile in-vivo body sensor networks," in Proceedings of the 3rd International Conference on Ubiquitous and Future Networks (ICUFN '11), pp. 95-100, Dalian, China, June 2011.

[52] IEEE Standard, IEEE Standard for Part 15 . 4: Wireless Medium Access Control (MAC) and Physical Layer (PHY) Specifications for Low-Rate Wireless Personal Area Networks (WPANs), IEEE Standard, 2006.

[53] C. Buratti, A. Conti, D. Dardari, and R. Verdone, "An overview on wireless sensor networks technology and evolution," Sensors, vol. 9, no. 9, pp. 6869-6896, 2009.

[54] B. Braem, B. Latré, I. Moerman, C. Blondia, and P. Demeester, "The wireless autonomous spanning tree protocol for multihop wireless body area networks," in Proceedings of the 3rd Annual International Conference on Mobile and Ubiquitous Systems: Networking \& Services, pp. 1-8, IEEE, San Jose, Calif, USA, July 2006.

[55] B. Latré, B. Braem, I. Moerman et al., "A low-delay protocol for multihop wireless body area networks," in Proceedings of the 4th Annual International Conference on Mobile and Ubiquitous Systems: Networking and Services (MobiQuitous '07), Philadelphia, Pa, USA, August 2007.

[56] A. G. Ruzzelli, R. Jurdak, G. M. P. O’Hare, and P. Van Der Stok, "Energy-efficient multi-hop medical sensor networking," in Proceedings of the 1st ACM SIGMOBILE International Workshop on Systems and Networking Support for Healthcare and Assisted Living Environments (HealthNet '07), pp. 37-42, ACM, San Juan, Puerto Rico, June 2007.

[57] A. G. Ruzzelli, G. M. P. O’Hare, M. J. O’Grady, and R. Tynan, "MERLIN: a synergetic integration of MAC and routing protocol for distributed sensor networks," in Proceedings of the $3 \mathrm{rd}$ Annual IEEE Communications Society on Sensor and Ad Hoc Communications and Networks, pp. 266-275, IEEE, Reston, Va, USA, September 2006.

[58] A. Bag and M. A. Bassiouni, "Biocomm-a cross-layer medium access control (MAC) and routing protocol co-design for biomedical sensor networks," International Journal of Parallel, Emergent and Distributed Systems, vol. 24, no. 1, pp. 85-103, 2009.

[59] N. Ababneh, N. Timmons, and J. Morrison, "Cross-layer optimization protocol for guaranteed data streaming over Wireless Body Area Networks," in Proceedings of the 8th IEEE International Wireless Communications and Mobile Computing Conference (IWCMC '12), pp. 118-123, IEEE, Limassol, Cyprus, August 2012.

[60] Y. Koucheryavy, L. Mamatas, I. Matta, and V. Tsaoussidis, Wired/Wireless Internet Communication, vol. 7277 of Lecture Notes in Computer Science, Springer, Berlin, Germany, 2012.

[61] H. Hwang, J. Lee, Y.-J. Hong, C. Bae, and N. M. Kim, "PARQ: controllable PLL ARQ method for wireless low power communication," in Proceedings of the IEEE 10th Consumer Communications and Networking Conference (CCNC '13), pp. 540-545, Las Vegas, Nev, USA, January 2013.

[62] N. Javaid, Z. Abbas, M. S. Fareed, Z. A. Khan, and N. Alrajeh, "M-ATTEMPT: a new energy-efficient routing protocol for wireless body area sensor networks," Procedia Computer Science, vol. 19, pp. 224-231, 2013.

[63] A. Ahmad, N. Javaid, U. Qasim, M. Ishfaq, Z. A. Khan, and T. A. Alghamdi, "RE-ATTEMPT: a new energy-efficient routing protocol for wireless body area sensor networks," International
Journal of Distributed Sensor Networks, vol. 2014, Article ID 464010, 9 pages, 2014.

[64] D. Djenouri and I. Balasingham, "New QoS and geographical routing in wireless biomedical sensor networks," in Proceedings of the 6th International Conference on Broadband Communications, Networks and Systems (BROADNETS '09), Madrid, Spain, September 2009.

[65] M. M. Monowar, M. Mehedi Hassan, F. Bajaber, M. A. Hamid, and A. Alamri, "Thermal-aware multiconstrained intrabody QoS routing for wireless body area networks," International Journal of Distributed Sensor Networks, vol. 2014, Article ID 676312, 14 pages, 2014.

[66] U. F. Abbasi, A. Awang, and N. H. Hamid, "A cross-layer opportunistic MAC/routing protocol to improve reliability in WBAN," in Proceedings of the Asia-Pacific Conference on Communications (APCC '14), pp. 36-41, IEEE, Pattaya, Thailand, October 2014.

[67] M. Monowar and F. Bajaber, "On designing thermal-aware localized QoS routing protocol for in-vivo sensor nodes in wireless body area networks," Sensors, vol. 15, no. 6, pp. 1401614044, 2015.

[68] J. Elias, "Optimal design of energy-efficient and cost-effective wireless body area networks," Ad Hoc Networks, vol. 13, pp. 560$574,2014$. 

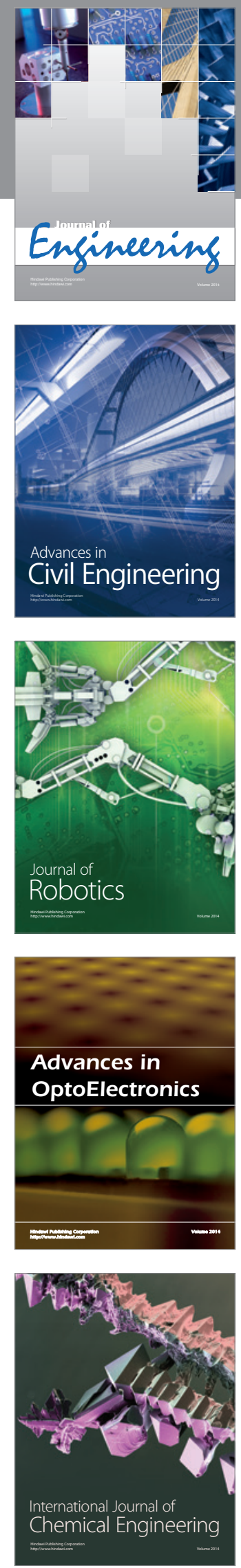

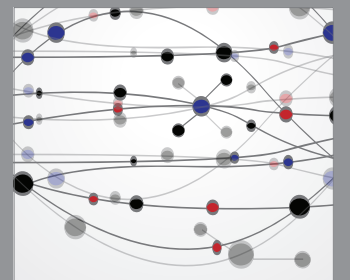

The Scientific World Journal
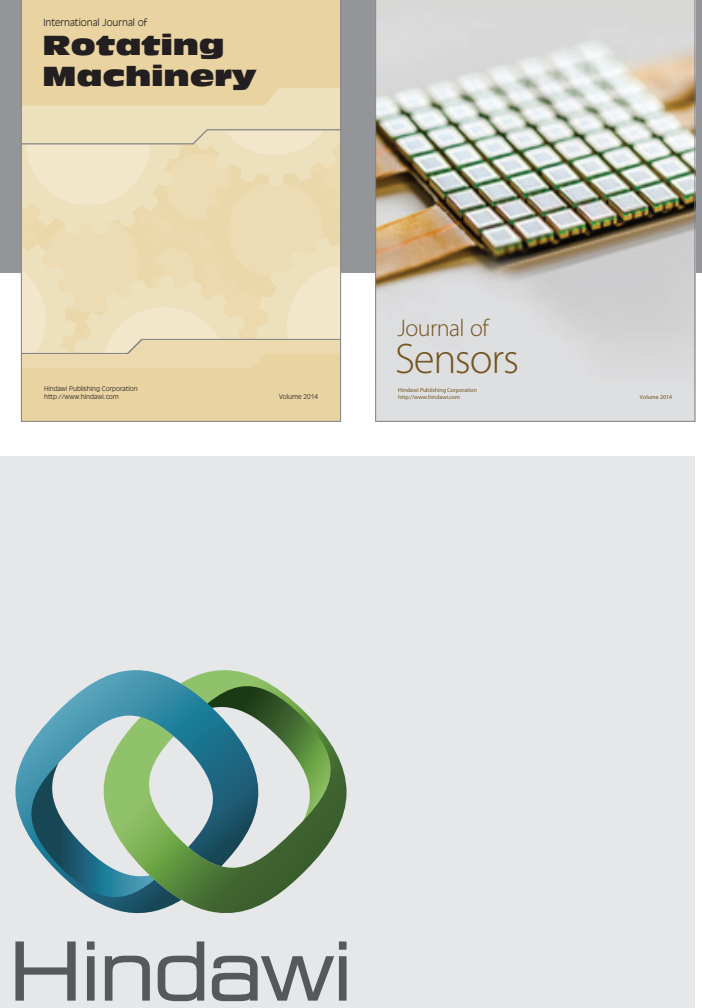

Submit your manuscripts at http://www.hindawi.com
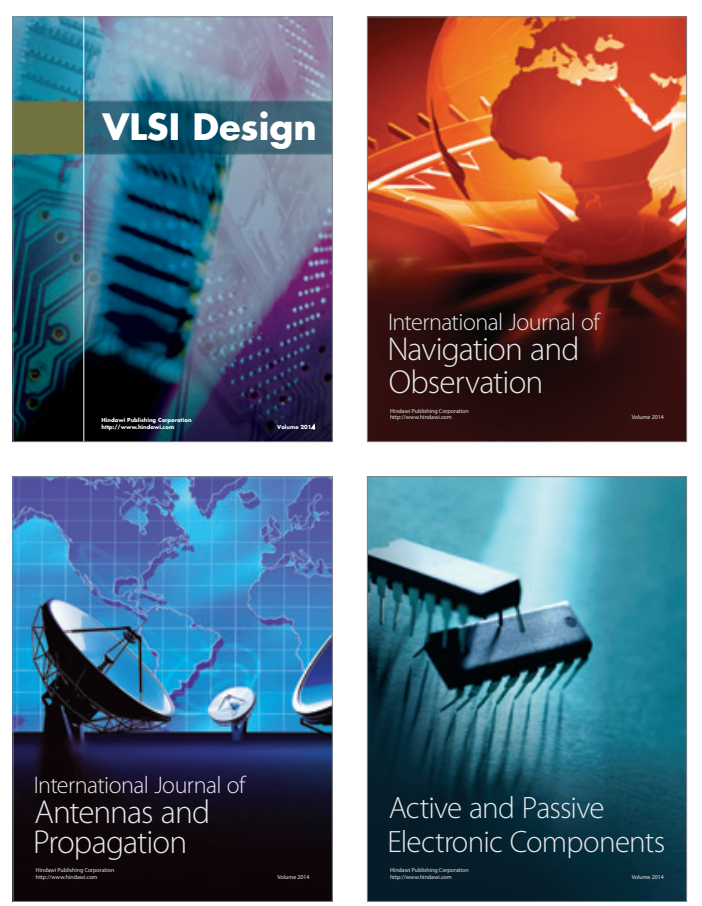
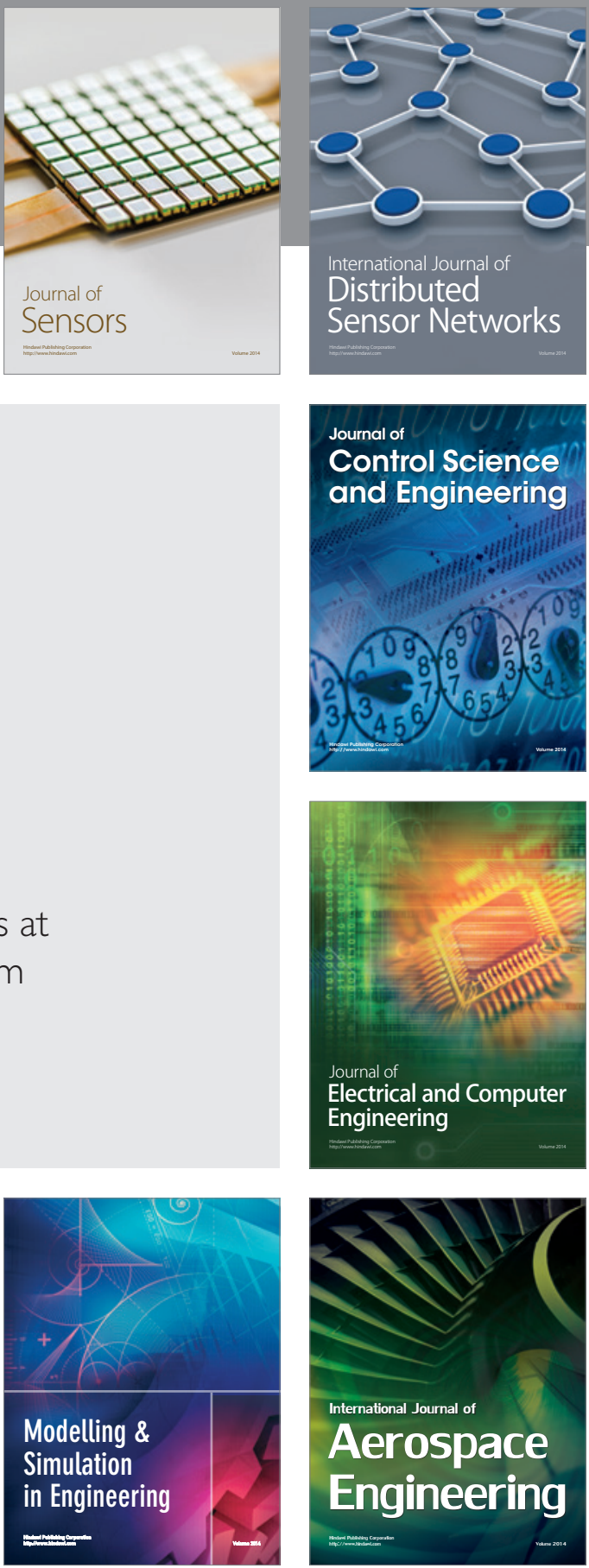

Journal of

Control Science

and Engineering
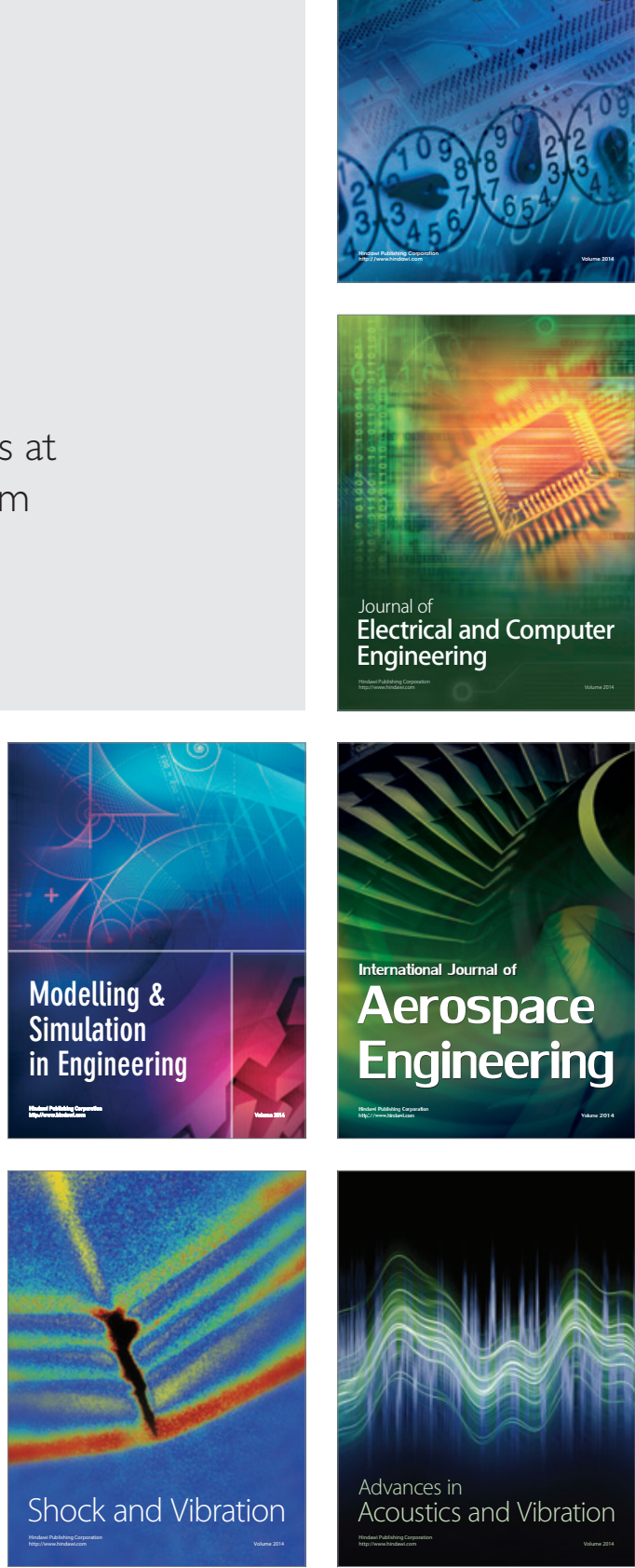